\title{
THE UNITARY REPRESENTATIONS OF THE GENERALIZED LORENTZ GROUPS
}

\author{
BY
}

\author{
ERNEST A. THIELEKER
}

ABSTRACT. For $n \geqslant 2$, let $G(n)$ denote the two-fold covering group of SO $_{e}(1, n)$. In case $n \geqslant 3, G(n)$ is isomorphic to $\operatorname{Spin}(1, n)$ and is simply connected. In a previous paper we determined all the irreducible quasi-simple representations of these groups, up to infinitesimal equivalence. The main purpose of the present paper is to determine which of these representations are unitarizable. Thus, with the aid of some results of Harish-Chandra and Nelson we determine all the irreducible unitary representations of $G(n)$, up to unitary equivalence. One by-product of our analysis is the explicit construction of the infinitesimal equivalences, which are known to exist from our previous work, between the various subquotient representations and certain subrepresentations in the nonirreducible cases of the nonunitary principal series representations of $G(n)$.

1. Introduction. For $n \geqslant 2$, let $G(n)$ denote a two-fold covering group of $\mathrm{SO}_{e}(1, n)$. The latter group is the identity component of the orthogonal group of a real nondegenerate quadratic form of signature $(+,-\cdots-)$ and of dimension $n+1$. In case $n \geqslant 3, G(n)$ is isomorphic to $\operatorname{Spin}(1, n)$ and is simply connected. For $n=2, G(n)$ is isomorphic to $\operatorname{SL}(2, \mathbf{R})$. In a previous paper we determined, up to infinitesimal equivalence, all the irreducible quasi-simple representations of $G(n)$. The main purpose of the present paper is to determine which of these representations are unitarizable. Thus, with the aid of some results of Harish-Chandra [3a, b] and Nèlson [6], we determine all the unitary representations of $G(n)$, up to unitary equivalence.

Before explaining the results and methods of this paper in more detail, we make some remarks concerning the notation. For any unexplained notation we refer the reader to [10]. One major change, however, is that $G(n)$ refers here to the group $\operatorname{Spin}(1, n)$, rather than to the group $\mathrm{SO}_{e}(1, n)$. A similar change in

Presented to the Society, in part, January 14, 1974 under the title On the explicit determination of Kunze-Stein intertwining operators with applications; received by the editors November 10, 1972.

Key words and phrases. Generalized Lorentz groups, unitary representations; intertwining operators, nonunitary principal series, universal enveloping algebras, modules over universal enveloping algebras, complementary series, classification of irreducible unitary representations.

Copyright $\odot 1974$, American Mathematical Society 
notation applies to the various subgroups of $\operatorname{Spin}(1, n)$ under consideration. In dealing with the nonunitary principal series representations here, it will be more convenient to use the so-called compact picture, rather than the induced picture extensively used in [10]. Thus, let $[H, \mu]$ be an irreducible finite-dimensional representation of the subgroup $M(n)$, and let $\Lambda$ be a complex character of the subgroup $S(n)$. Then $\Lambda \mu$ is a finite-dimensional irreducible representation of the parabolic subgroup $S(n) M(n)$. Let $\rho_{K}$ denote the restriction-to- $K(n)$ map which takes functions in the space $C_{\Lambda \mu}(G(n), H)$ onto their restrictions to $K(n)$. Then $\rho_{K}$ is a linear isomorphism, and a $K(n)$-module isomorphism from the $K(n)$-module $\left[C_{\Lambda \mu}(G(n), H), R\right]$ to the $K(n)$-module $\left[C_{\mu}(K(n), H), R\right]$. Hence $\rho_{K}$ extends to an isometry $\hat{\rho}_{K}$ from the Hilbert space completion $L_{\Lambda \mu}^{2}(G(n), H)$ to the Hilbert space completion $L_{\mu}^{2}(K(n), H)$. As in $[10, \S 8]$ we denote by $\Pi_{\Lambda}$ the action of $G(n)$ on $L_{\mu}^{2}(K(n), H)$ defined by $\Pi_{\Lambda}(g)=\hat{\rho}_{K}(g) R(g) \hat{\rho}_{K}^{-1}$ for all $g \in G(n)$. As in [10], let $\left[d C_{\mu}(K(n), H), d \Pi_{\Lambda}\right]$ denote the $U(\mathbf{G}(n))$-module of $K(n)$-finite vectors of $\left[L_{\mu}^{2}(K(n), H), \Pi_{\Lambda}\right]$. (Of course, this $U(\mathrm{G}(n))$-module is also the $U(\mathbf{G}(n))$-module of $K(n)$-finite vectors for the Banach space representation $\left[C_{\mu}(K(n), H), \Pi_{\Lambda}\right]$.)

By using some results of [10], in particular Theorem 1 of that paper, we construct the possible $U(\mathbf{G}(n))$-module homomorphisms (intertwining maps) from the $U(\mathbf{G}(n))$-module $\left[d C_{\mu}(K(n),+), d \Pi_{\Lambda}\right]$ to the $U(\mathbf{G}(n))$-module $\left[d C_{-\mu}^{-}(K(n), H)\right.$, $\left.d \Pi_{\Lambda^{\prime}}\right]$, where $\bar{\mu}$ is the irreducible representation of $M(n)$ corresponding to the character of $M(n)$ which is complex conjugate to the character of $\mu . \Lambda^{\prime}$ is the Weyl conjugate character of $S(n)$ defined by $\Lambda^{\prime}=\Lambda^{-1} \mathrm{P}^{2}$, where we recall that $\mathrm{P}^{2}$ is the character of $S(n)$ defined by $\mathrm{P}^{2}(s)=\operatorname{det} \operatorname{Adl}_{S(n)}(s)$, for $s \in$ $S(n)$. Equivalently, $\mathrm{P}^{2}$ is determined by the condition $d \mathrm{P}^{2}(H)=2 d \mathrm{P}(H)=$ $(n-1)$. Note also that $\bar{\mu}$ is the representation of $M(n)$ which is conjugate to $\mu$ by the automorphism of $M(n)$ determined by the nontrivial element of the Weyl group of $G(n) / K(n)$. Similarly, $\Lambda^{\prime}$ is the conjugate of $\Lambda$ determined by this Weyl group element in the usual way. In more detail, we use the formula in Theorem 1 of [10] to obtain a recursion relation for the Fourier components of the intertwining operators. These recursion relations can be solved completely in terms of certain rational functions of $d \Lambda(H)$ (essentially ratios of $\Gamma$-functions). It follows, incidentally, from our main result that the $U(\mathrm{G}(n))$-module homomorphisms constructed here must be proportional to the restrictions to the space of $K(n)$-finite vectors of the integral operators of Knapp and Stein [5], or of those of Schiffmann [8], in the cases where these integral operators are defined. We point out however that our methods yield intertwining operators for all characters $\Lambda$, even for those which correspond to the poles of the meromorphically continued Knapp and Stein operators. For these "singular" characters $\Lambda$ our intertwining homomorphisms have nontrivial kernels. Thus one by-product of our analysis is to 
construct explicitly the isomorphisms which exist between quotient representations and certain subrepresentations in the nonirreducible cases of the nonunitary principal series. The existence of these isomorphisms was pointed out by us in [10] by using some results on characters of Harish-Chandra.

Because of the explicit form of the $U(\mathbf{G}(n))$-module homomorphisms constructed here, it is relatively easy to determine which of these homomorphisms lead to positive definite inner products. By using these results, we get a complete classification of the infinitesimally unitary representations. By using a result of Nelson [6], one can then get a complete classification of the irreducible unitary representations of $G(n)$. However, in the case of the complementary series that Knapp and Stein get for $G(n)$, we can construct such a globalization explicitly.

Although the details of the results on unitary representations appear in $\$ 7$, let us make some general remarks on these results. For the purposes of discussing unitary representations, it is convenient to use the complex parameter $v$ defined by $\nu=d \Lambda(H)+(n-1) / 2$. Then the principal series correspond to imaginary $\nu$. In addition, there are certain complementary series corresponding to certain overlapping intervals on the real $\nu$-axis. These have been constructed by Knapp and Stein in [5] for the groups under consideration here, as well as for the other split-rank one classical groups. In addition to the complementary series there are also unitary representations corresponding to the right end points of the Knapp and Stein complementary series intervals, as well as unitary representations corresponding to certain isolated points on the real $\nu$-axis. These end point and isolated point representations correspond to some of the nonirreducible cases of the nonunitary principal series. For the latter unitary representations the intertwining operators define positive (indefinite) inner products on the spaces of $K(n)$ finite vectors. The radicals of these forms are just the kernels of the corresponding intertwining homomorphisms, and the corresponding unitary representations can be defined on the Hilbert space completions of the quotients of the induced representations modulo these kernels. It is known that in the special case of SL $(2, \mathbf{R})$, the discrete series representations arise in this manner. This fact can easily be established for this group by the computations of Bargmann [1]. In the general case, however, the kernels are not finite dimensional, in general. For $n=4$, the square integrable representations of Dixmier can also be obtained in this way. A formal analogy with the Dixmier representations allows us to guess which of these isolated point representations correspond to square integrable representations in the case of general even $n$. However, we do not prove square integrability of these representations in this paper.

The following is an outline of the paper. In the next section we derive the recursion relations for the Fourier components of the intertwining operators. In $\S 3$ we construct the various candidates for these intertwining operators and state 
the main result for them (Theorem 1). In $\S 4$ this theorem is proved, along with some lemmas which are also needed for the remaining results of the paper. We remark incidentally that the form of these Fourier components of the intertwining operators reduce in the case of class 1 representations, to some formulas for Fourier components of intertwining operators announced by Johnson and Wallach in [4]. However, Wallach's formulas are valid for more general real-rank 1 groups. (See also [11].) In $\$ 5$ we apply the results on the intertwining maps to obtain an explicit construction of the isomorphisms between the subquotient representations in the nonirreducible cases of the nonunitary principal series, and certain subrepresentations. These isomorphisms were already known to exist from our work in [10], but there we based our argument on some results of Harish-Chandra's character theory. In $\S 6$ we discuss infinitesimally pseudounitary representations as a preliminary step in the discussion of the unitary representations. The unitary representations are classified in $\S 7$, and the results of the arguments in that section are summarized in Theorem 3. In $\S 8$ we compare our results with some known results and offer some conjectures.

We gratefully acknowledge some encouraging comments made by $\mathrm{N}$. Wallach concerning the methods and the problems under consideration in this paper.

\section{Basic recursion relations for the Fourier components of the intertwining} operators. We make some preliminary remarks and recall some notation from [10]. If $L$ denotes a compact group, then $\Omega(L)$ denotes the set of equivalence classes of finite-dimensional irreducible representations of $L$. Of course, this set is equal to the set of equivalence classes of irreducible unitary representations of L. If $[\mu] \in \Omega(M(n))$, we denote by $\Omega_{\mu}(K(n))$ the set of classes in $\Omega(K(n))$ whose restrictions to the subgroup $M(n)$ contain the class $[\mu]$, under complete reduction. The elements of the subset $\Omega_{\mu}(K(n))$ are called the $\mu$-admissible classes in $\Omega(K(n))$. As in $[10, \S 3]$, we define an Hermitian inner product on $\mathbf{G}(n)_{\mathbf{C}}$, given by $(X, Y)=-c B(X, \Theta Y)$, for $X$ and $Y \in \mathbf{G}(n)$, and where $B$ is the Killing form on $\mathbf{G}(n)_{\mathbf{C}}$, and $\Theta$ is the Cartan involution corresponding to the Cartan decomposition $\mathbf{G}(n)=\mathbf{K}(n)+\mathbf{P}(n)$, and $c=1 / 2(n-1)$. Then let $H$ denote the element of $\mathrm{P}(n)$ given in matrix units by $H=Y_{01}=E_{01}+E_{10}$; so that we may, and do, take $\mathbf{A}(n)=\mathbf{R} H$. Then also $\alpha(H)=1$, where $\alpha$ is the unique restricted positive root with respect to some Weyl chamber in $\mathbf{A}(n)$. We let $\mathbf{H}(n)$ be some Cartan subalgebra of $\mathbf{K}(n)$, such that the intersection $\mathbf{H}(n) \cap \mathbf{M}(n)$ is a Cartan subalgebra of the subgroup $M(n)$. Let $p$ be the rank of $\mathbf{K}(n)$, and let $\left(i \epsilon_{1}, \cdots, i \epsilon_{p}\right)$ be an ordered orthonormal basis of $\mathbf{H}(n)_{\mathbf{C}}$; so that $\epsilon_{i}, 1 \leqslant i \leqslant p$, are real forms on $i \mathrm{H}(n)$. (We identify $\mathrm{H}(n)_{\mathrm{C}}$ with its dual via the form $($,$) ).) Then, for each class [\omega] \in \Omega(K(n))$, the highest weight $\Lambda_{\omega}$ can be expressed in the form $\Lambda_{\omega}=\Sigma_{1}^{p} \Lambda_{\omega i} \epsilon_{i}$, where the components 
$\Lambda_{\omega i}$ are all integers or half odd integers which satisfy the inequalities: $\Lambda_{\omega 1} \geqslant$ $\Lambda_{\omega 2} \geqslant \cdots \geqslant\left|\Lambda_{\omega p}\right|$ in case $n=2 p$, and $\Lambda_{\omega 1} \geqslant \Lambda_{\omega 2} \geqslant \cdots \geqslant \Lambda_{\omega p} \geqslant 0$, in case $n=2 p+1$. When $n=2 p \geqslant 4$, we chose the basis $\left(i \epsilon_{1}, \cdots, i \epsilon_{p}\right)$ such that $\left(i \epsilon_{1}, \cdots, i \epsilon_{p-1}\right)$ is an ordered basis of $\mathbf{H}(n) \cap \mathbf{M}(n)$. (In case $n=$ $2 p+1, \operatorname{rank}(\mathbf{M}(n))=p$; so that $\mathbf{M}(n) \supset \mathbf{H}(n)$.) When it is convenient to do so, we identify the elements of $\Omega(K(n))$ with their highest weights.

Let us realize the Weyl group of $G(n) / K(n)$ as the factor group $N_{K}(M(n)) / M(n)$, where $N_{K}(M(n))$ is the normalizer of $M(n)$ in $K(n)$. Then there exists an element $\bar{w}$ in the coset other than $M(n)$ such that $\operatorname{Ad}(\bar{w}) \epsilon_{i}=\epsilon_{i}$, for $i \neq$ $p$, and $\operatorname{Ad}(\bar{w}) \epsilon_{p}=-\epsilon_{p}$. It follows easily that if $n=2 p$ and if $[\mu] \in \Omega(M(n))$, then the Weyl conjugate representation: $m \rightarrow \mu^{w}(m)=\mu(\operatorname{Ad}(\bar{w}) m)$ is equal to $\mu$, while for $n=2 p+1$, and $[\mu] \in \Omega(M(n))$, the Weyl conjugate representation $\mu^{w}$ has highest weight $\Lambda_{\mu} w_{i}$ whose components are $\Lambda_{\mu} w_{i}=\Lambda_{\mu i}$ for $i \neq p$, and $\Lambda_{\mu} w_{p}=-\Lambda_{\mu p}$. Thus this Weyl conjugate is equal to the representation $\bar{\mu}$ whose character is the complex conjugate of the character of $\mu$. We note finally that $\operatorname{Ad}(\bar{w}) H=-H$.

As in [10] we define for each $Y \in \mathbf{P}(n)$ the function $\Phi_{Y}$ on the subgroup $K(n)$ by $k \rightarrow \Phi_{Y}(k)=(\operatorname{Ad}(k) Y, H)$, and let $S$ denote the pointwise algebra of functions generated over $\mathbf{C}$ by these functions. Then $S$ is the pointwise algebra generated by the polynomial functions on the sphere (Ad $K) h$.

Now let $T$ be a $U(\mathbf{G}(n))$-module homomorphism from the $U(\mathbf{G}(n))$ module $\left[d C_{\mu}(K(n), H), d \Pi_{\Lambda}\right]$ to the $U(\mathbf{G}(n))$-module $\left[d C_{\bar{\mu}}(K(n), H), d \Pi_{\Lambda^{\prime}}\right]$, where for each complex character $\Lambda(S(n)), \Lambda^{\prime}$ denotes the character $\Lambda^{-1} \mathrm{P}^{2}$. We also write $\nu=d \Lambda(H)-d P(H)=\lambda-(n-1) / 2$. Then we have for the parameter $\nu^{\prime}$, defined by $\nu^{\prime}=d \Lambda^{\prime}(H)-d P(H), \nu^{\prime}=-\nu$. We remark that $\mu=\bar{\mu}$, if and only if $n=2 p$ or $n=2 p+1$ and the component $\Lambda_{\mu p}$ is equal to zero. More generally, let $U$ be any intertwining map from the $U(\mathbf{G}(n))$-module $\left[d C_{\mu}(K(n), H), d \Pi_{\Lambda}\right]$ to the $U(\mathbf{G}(n))$-module $\left[d C_{\mu^{\prime \prime}}\left(K(n), H^{\prime \prime}\right), d \Pi_{\Lambda^{\prime \prime}}\right]$, where $\left[H^{\prime \prime}, \mu^{\prime \prime}\right]$ is not necessarily equal to $[H, \bar{\mu}]$, and $\Lambda^{\prime \prime}$ is not necessarily equal to $\Lambda^{\prime}$. Let $E_{\omega}$ denote, for each class $[\omega] \in \Omega(K(n))$, the Hermitian projection on the Hilbert space direct sum: $\bar{\bigoplus}\left\{L_{\tau}^{2}\left(K(n), H_{\tau}\right):[\tau] \in \Omega(M(n))\right\}$ which projects onto the subspace of elements which transform under $[\omega]$. (For any class $[\tau]$, $L_{\tau}^{2}\left(K(n), H_{\tau}\right)$ is identified with its canonical projection in this direct sum.) Since $U$ intertwines the action of right translations by elements of $K(n)$, it follows from a standard argument that $U$ commutes with the operator $E_{\omega}$ for each class $[\omega] \in \Omega(K(n))$. We denote by $U_{\omega}$ the operator $U_{\omega}=E_{\omega} U=U E_{\omega}$, which maps $L_{\mu}^{2}(K(n), H)$ into $L_{\mu}^{2 \prime}\left(K(n), H^{\prime \prime}\right)$. For each class $[\omega] \in \Omega(K(n))$, $U_{\omega}$ is called the Fourier component of $U$ belonging to the class [ $\omega$ ]. Clearly, by the Frobenius reciprocity theorem for compact groups, $U_{\omega}=0$, if $[\omega]$ is not $\mu$-admissible. 
Now, let us assume that $\left[H^{\prime \prime}, \mu^{\prime \prime}\right]=[H, \mu]$. Then $U$ must intertwine the action of $K(n)$ on $d C_{\mu}(K(n), H)$, which is the action of right translations. It follows easily from Schur's lemma, and from the fact that each class in $\Omega(K(n))$ occurs with multiplicity at most one in the complete reduction of the $K(n)$ module $L_{\mu}^{2}(K(n), H)$, that $U_{\omega}=u_{n}(\mu, \nu, \omega) E_{\omega}$, where $u_{n}(\mu, \nu, \omega)$ is a complex number which is different from zero only if $[\omega]$ is $\mu$-admisssible. If $\mu=$ $\bar{\mu}$, then we can similarly write for the Fourier components of $T, T_{\omega}=$ $t_{n}(\mu, \nu, \omega) E_{\omega}$, where $t_{n}(\mu, \nu, \omega)$ is a complex number, which is different from zero only if $[\omega]$ is a $\mu$-admissible class.

The first lemma applies to the situation when $\mu=\bar{\mu}$. We write $\nu^{\prime \prime}=$ $d \Lambda(H)-(n-1) / 2$.

LEMmA 1 (BASIC RECURSION RELATION). Assume that for some index $i$ with $1 \leqslant i \leqslant p$, that $\Lambda_{\omega}$ and $\Lambda_{\omega}+\epsilon_{i}$ are both highest weights of $\mu$-admissible classes in $\Omega(K(n))$. Then the following statements are true.

(1) The numbers $u_{n}\left(\mu, \nu, \Lambda_{\omega}\right)$, and $u_{n}\left(\mu, \nu, \Lambda_{\omega}+\epsilon_{i}\right)$ must satisfy the pair of equations (here we identify the elements of $\Omega(K(n)$ ) with their highest weights):

$$
\begin{aligned}
& \left(\nu^{\prime \prime}+\Lambda_{\omega i}-i+(n+1) / 2\right) u_{n}\left(\mu, \nu, \Lambda_{\omega}\right)-\left(\nu+\Lambda_{\omega i}-i+(n+1) / 2\right) u_{n}\left(\mu, \nu, \Lambda_{\omega}+\epsilon_{i}\right)=0, \\
& \left(\nu-\Lambda_{\omega i}+i-(n+1) / 2\right) u_{n}\left(\mu, \nu, \Lambda_{\omega}\right)-\left(\nu^{\prime \prime}-\Lambda_{\omega i}+i-(n+1) / 2\right) u_{n}\left(\mu, \nu, \Lambda_{\omega}+\epsilon_{i}\right)=0 .
\end{aligned}
$$

(2) The map $U$ is nonzero only if either $\nu^{\prime \prime}=-\nu$, or $\nu^{\prime \prime}=\nu$. In the latter case $U$ is a scalar map.

(3) Let $T$ be an intertwining map from $\left[d C_{\mu}(K(n), H), d \Pi_{\Lambda}\right]$ to $\left[d C_{\mu}(K(n), H), d \Pi_{\Lambda^{\prime}}\right]$. Then the complex coefficients $t_{n}\left(\mu, \nu, \Lambda_{\omega}+\epsilon_{i}\right)$ must satisfy the recursion relations

$$
\begin{aligned}
\left(\Lambda_{\omega i}-i+(n+1) / 2-\nu\right) t_{n}\left(\mu, \nu, \Lambda_{\omega}\right) & \\
& =\left(\Lambda_{\omega i}-i+(n+1) / 2+\nu\right) t_{n}\left(\mu, \nu, \Lambda_{\omega}+\epsilon_{i}\right) .
\end{aligned}
$$

Proof. Let $\left[\omega^{\prime}\right]$ denote the class in $\Omega(K(n))$ corresponding to the highest weight $\Lambda_{\omega}+\epsilon_{i}$. It follows from Theorem 1 in [10], and from the explicit calculation in the proof of Theorem 2 in [10], that for all elements $Y \in P(n)_{\mathbf{C}}$, and $f \in E_{\omega} L_{\mu}^{2}(K(n), H)$ we have

$$
E_{\omega^{\prime}} d \Pi_{\Lambda}(Y) f=\left(\lambda+\Lambda_{\omega i}+1-i\right) E_{\omega^{\prime}} \Phi_{Y} f
$$

where $\lambda=d \Lambda(H)$. From the same results we also have for $f^{\prime} \in E_{\omega}{ }^{\prime} L_{\mu}^{2}(K(n), H)$,

$$
E_{\omega} d \Pi_{\Lambda}(Y) f^{\prime}=\left(\lambda-\Lambda_{\omega i}-n+i\right) E_{\omega} \Phi_{Y} f^{\prime} .
$$

Now set $\lambda^{\prime \prime}=d \Lambda^{\prime \prime}(H)$, apply $U$ to equation (a) and use the intertwining property of $U$. We then obtain: 


$$
\begin{array}{r}
u_{n}\left(\mu, \nu, \Lambda_{\omega}+\epsilon_{i}\right)\left(\lambda+\Lambda_{\omega i}+1-i\right) E_{\omega^{\prime}} \Phi_{Y} f=E_{\omega^{\prime}} U d \Pi_{\Lambda}(Y) f=E_{\omega^{\prime}} d \Pi_{\Lambda^{\prime \prime}}(Y) U f \\
\quad=u_{n}\left(\mu, \nu^{\prime \prime}, \Lambda_{\omega}\right) E_{\omega^{\prime}} d \Pi_{\Lambda^{\prime \prime}}(Y) f=u_{n}\left(\mu, \nu^{\prime \prime}, \Lambda_{\omega}\right)\left(\lambda^{\prime \prime}+\Lambda_{\omega i}+1-i\right) E_{\omega^{\prime}} \Phi_{Y} f
\end{array}
$$

where in the last step, formula (a) is used again with $\lambda$ replaced by $\lambda^{\prime \prime}=d \Lambda^{\prime \prime}(H)$.

Now, if $f \neq 0$, then for some element $Y \in \mathrm{P}(n)$ we must have $E_{\omega^{\prime}} \Phi_{Y} f$ $\neq 0$. Assume indirectly that $E_{\omega^{\prime}} \Phi_{Y} f=0$ for all $Y \in \mathrm{P}(n)$. By taking linear combinations of right translates by elements of $K(n)$, it follows that $E_{\omega^{\prime}} \Phi_{Y} F_{\omega}=$ 0 , where $F_{\omega}$ is the subspace given by $F_{\omega}=E_{\omega} L_{\mu}^{2}(K(n), H)$, which by hypothesis is different from zero. Let $D$ denote the projection given by $D=E_{\omega}+$ $E_{\omega^{\prime}}$. This projection satisfies the hypothesis of Lemma 5 in [10]. Hence by that lemma we have $D L_{\mu}^{2}(K(n), H)=D S F_{\omega}=S_{D} F_{\omega}$, where $S_{D}$ is the operator algebra generated by the operators $D \Phi_{Y}$, with $Y \in \mathrm{P}(n)$. On the other hand, by the indirect hypothesis we have $D \Phi_{Y} F_{\omega}=F_{\omega}$. It follows by an induction argument based on the filtration of $S_{D}$ that $S_{D} F_{\omega}=F_{\omega}$. This result leads to a contradiction, since by hypothesis of the lemma, $D F_{\omega} \neq F_{\omega}$. Thus the assertion is proved.

The first equation of the lemma now follows immediately by the substitution: $\nu=\lambda-(n-1) / 2$, and $\nu^{\prime \prime}=\lambda^{\prime \prime}-(n-1) / 2$. By applying an argument similar to the above one to equation (b), we obtain the second equation. Thus statement 1 is proved.

The conditions $\nu=\nu^{\prime \prime}$, or $\nu=-\nu^{\prime \prime}$ in statement 2 result from the usual determinant condition for the existence of nontrivial solutions of a linear system. First, assume that $\nu^{\prime \prime}=\nu$. Then both of these equations of statement 1 result in the equation $u_{n}\left(\mu, \nu, \Lambda_{\omega}\right)=u_{n}\left(\mu, \nu, \Lambda_{\omega}+\epsilon_{i}\right)$. Assume that $U \neq 0$. Then $u_{n}\left(\mu, \nu, \Lambda_{\omega}\right) \neq 0$ for some class $[\omega] \in \Omega_{\mu}(K(n))$. Now, apply induction in each of the $p$-directions $\epsilon_{i}$, with $1 \leqslant i \leqslant p$. Thus, we find that $u_{n}(\mu, \nu, \omega)=$ $u_{n}(\mu, \nu, \tau)$ for all classes $[\tau] \in \Omega_{\mu}(K(n))$. Hence the second statement follows.

Statement 3 follows by the substitution $\nu^{\prime \prime}=-\nu$ in either of the two equations in statement 1. Q.E.D.

Next, we assume that the character of $\mu$ is not real, so that $\mu \neq \bar{\mu}$. As pointed out above, this situation only occurs when $n=2 p+1$, and the component $A_{\mu p}$ is different from zero. In order to deal with this situation we must modify the considerations connected with Lemma 1. For this case the unitary induced representation theory of semidirect product groups is helpful.

LEMma 2. (1) For $n \geqslant 2$, there exists an isometry I from the Hilbert space $L_{\mu}^{2}(K(n), H)$ to the Hilbert space $L_{\mu}^{2}(K(n), H)$ such that $I$ intertwines multiplication by $\Phi_{Y}$, for all $Y \in \mathbf{P}(n)$ as follows. Let $f \in d C_{\mu}(K(n), H)$. Then

$$
I \Phi_{Y} f=-\Phi_{Y} I f
$$


Moreover, $I$ intertwines the action of right translations by elements of $K(n)$.

(2) Assume that $n \neq 2 p+1$ and that $\bar{\mu} \neq \mu$ Let $T$ be a $U(\mathbf{G}(n))$ module homomorphism from $\left[d C_{\mu}(K(n), H), d \Pi_{\Lambda}\right]$ into $\left[d C_{\bar{\mu}}(K(n), H), d \Pi_{\Lambda^{\prime}}\right]$. Assume that $\Lambda_{\omega}$ and $\Lambda_{\omega}+\epsilon_{i}$, for some $i$, with $1 \leqslant i \leqslant p$, are both highest weights of $\mu$-admissible classes in $\Omega(K(n))$. Then if I denotes an isometry whose existence is assured by statement 1 and which has the properties stated there, then the Fourier components $T_{\omega}$ and $T_{\Lambda_{\omega}+\epsilon_{i}}$ are given by

$$
T_{\omega}=w_{n}\left(\mu, \nu, \Lambda_{\omega}\right) E_{\omega} I \text {, and } T_{\Lambda_{\omega}+\epsilon_{i}}=w_{n}\left(\mu, \nu, \Lambda_{\omega}+\epsilon_{i}\right) E_{\Lambda_{\omega}+\epsilon} I \text {, }
$$

where $w_{n}\left(\mu, \nu, \Lambda_{\omega}\right)$ and $w_{n}\left(\mu, \nu, \Lambda_{\omega}+\epsilon_{i}\right)$ are complex numbers which satisfy the equation:

$$
\begin{aligned}
\left(\Lambda_{\omega i}-i\right. & +(n+1) / 2-\nu) w_{n}\left(\mu, \nu, \Lambda_{\omega}\right) \\
& =-\left(\Lambda_{\omega i}-i+(n+1) / 2+\nu\right) w_{n}\left(\mu, \nu, \Lambda_{\omega}+\epsilon_{i}\right) .
\end{aligned}
$$

Proof. Let $G_{0}$ denote the motion group associated with the Cartan decomposition $\mathbf{G}(n)=\mathbf{P}(n)+\mathbf{K}(n)$. Thus $G_{0}$ is isomorphic to a semidirect product of $K(n)$ and the vector addition group of $\mathrm{P}(n)$. Accordingly, the multiplication on $G_{0}$ can be given by:

$$
\left((X, k),\left(X^{\prime}, k^{\prime}\right)\right) \rightarrow\left(X+\operatorname{Ad}(k) X^{\prime}, k k^{\prime}\right)
$$

with $X, X^{\prime} \in \mathrm{P}(n)$, and $k, k^{\prime} \in K(n)$. For each element $Y \in \mathrm{P}(n)$ let $\tau_{Y}$ denote the character of $\mathrm{P}(n)$ defined by $\tau_{Y}\left(Y^{\prime}\right)=e^{i\left(Y^{\prime}, Y\right)}$. Since $\mathrm{P}(n)$ is a normal subgroup of $G_{0}, G_{0}$ acts on these characters in the usual way. In particular, if $k \in K(n), \tau_{Y}^{k}=\tau_{\mathrm{Ad}\left(k^{-1}\right) Y^{*}}$. Then $(\mathrm{P}(n), M(n))$, is the stabilizer subgroup of the character $\tau_{H}$ under this action. Moreover, the characters $\tau_{H}$ and $\tau_{-H}$ are conjugate under the Weyl group element $\bar{w}$. Let $\tau_{H} \mu$ denote the finite-dimensional irreducible representation of this stabilizer subgroup defined by $\left(\tau_{H} \mu\right)\left(Y^{\prime}, m\right)=\tau_{H}\left(Y^{\prime}\right) \mu(m)$, with $Y^{\prime} \in \mathbf{P}(n)$, and $m \in M(n)$. Similarly $\tau_{-H^{\prime}} \bar{\mu}$ denotes the finite-dimensional irreducible representation of this stabilizer subgroup defined by $\left(\tau_{-H} \bar{\mu}\right)\left(Y^{\prime}, m\right)=\tau_{-H}\left(Y^{\prime}\right) \bar{\mu}(m)$, for $Y^{\prime} \in \mathrm{P}(n)$, and $m \in M(n)$. These two representations are therefore conjugate under the action of the element $\bar{w}$. It follows from a celebrated theorem of Mackey [7, Theorem 14.1], that the unitary induced representations $U^{\tau} H^{\mu}$ and $U^{\tau}-H^{\bar{\mu}}$ are unitarily equivalent. Now, the representations $U^{\tau} H^{\mu}$ and $U^{\tau}-H^{\mu}$ may be considered as acting on the Hilbert spaces $L_{\mu}^{2}(K(n), H)$ and $L_{\bar{\mu}}^{2}(K(n), H)$, respectively. Let $I$ denote an isometry from the first to the second of these Hilbert spaces. The restriction to $K(n)$ of each of these two induced representations is the action of right translations by elements of $K(n)$, while the action of $\mathrm{P}(n)$ is given by

$$
U^{\tau} H^{\mu}(Y) f(k)=\tau_{H}(\operatorname{Ad}(k) Y) f(k) \quad \text { and } \quad U^{\tau}-H^{\mu}(Y) g(k)=\tau_{-H}(\operatorname{Ad}(k) Y) g(k) \text {, }
$$


for almost all $k \in K(n), f \in L_{\mu}^{2}(K(n), H), g \in L_{\bar{\mu}}^{2}(K(n)$, H), and $Y \in \mathbf{P}(n)$. By Mackey's theorem, we may assume that the isometry $I$ intertwines these actions. Hence,

$$
\left(I \tau_{H}(\operatorname{Ad}(\cdot) Y) f(k)=\tau_{-H}(\operatorname{Ad}(k) Y)(I f)(k),\right.
$$

for almost all $k \in K(n)$. Now restrict $f$ to lie in the linear subspace $d C_{\mu}(K(n), H)$. The latter subspace consists of differentiable, even real-analytic functions. Hence, by differentiating along the one-parameter subgroup of $\boldsymbol{G}_{\mathbf{0}}$ given by $t \rightarrow(t Y, e)$, statement 1 follows.

Now we make the assumptions of statement 2 in the lemma. Let $I_{\sigma}$ denote, for each class $[\sigma] \in \Omega(K(n))$, the Fourier component of $I$; that is, $I_{\sigma}=$ $E_{\sigma} I=I E_{\sigma}$. If $[\sigma]$ is a $\mu$-admissible class, then $I_{\sigma}$ is a one-to-one map from $E_{\sigma} L_{\mu}^{2}(K(n), H)$ to the subspace $E_{\sigma} L_{\bar{\mu}}(K(n), H)$. Let $I_{\sigma}^{-1}$ denote the inverse map of $I_{\sigma}$. Then both $I_{\sigma}$ and $I_{\sigma}^{-1}$ are $K(n)$-module isomorphisms. Hence the map $I_{\sigma}^{-1} T_{\sigma} I_{\sigma}$ is a linear transformation on the finite-dimensional space $E_{\sigma} L_{\mu}^{2}(K(n), H)$ which commutes with the action of $K(n)$. Since this action is irreducible, it follows from Schur's lemma that $I_{\sigma}^{-1} T_{\sigma} I_{\sigma}=t_{n}(\mu, \nu, \sigma) 1_{\sigma}$, where $1_{\sigma}$ is the identity map on $E_{\sigma} L_{\mu}^{2}(K(n), H)$ and where $t_{n}(\mu, \nu, \sigma)$ is a complex number. The remainder of the proof now follows the pattern of proof as Lemma 1 , with the exception of some obvious modifications, by applying the intertwining map $T$ to each of the equations (a) and (b) in the proof of Lemma 1. The details are left to the reader. Q.E.D.

Statement 1 of the last lemma has a useful corollary which we state as Lemma 3 below. This corollary has some implications for the theory of spherical functions on the $(n-1)$-sphere. For $n=3$, it is known, and "explains" the fact that these functions obey three-term recursion relations, rather than four-term recursion relations.

Lemma 3. Let $Y \in \mathrm{P}(n),[\omega] \in \Omega(K(n))$, and $f \in E_{\omega} L_{\mu}^{2}(K(n)$, H). If $\mu=\bar{\mu}$, then $E_{\omega} \Phi_{Y} f=0$.

Proof. If $[\omega]$ is not a $\mu$-admissible class, the result is immediate. Assume that $[\omega]$ is $\mu$-admissible. Since $\mu=\bar{\mu}$, the isometry in statement 1 of Lemma 2 must be a scalar. This fact follows, either from the irreducibility of the unitary representation $U^{\tau} H^{\mu}$ (another celebrated theorem of Mackey) or from the density of the linear subspace $S E_{\omega} L_{\mu}^{2}(K(n), H)$ (Lemma 5 of [10]). It follows that for some scalar $c$ with $|c|=1$, we have

$$
c E_{\omega} \Phi_{Y} f=I E_{\omega} \Phi_{Y} f=-E_{\omega} \Phi_{Y} I f=-c E_{\omega} \Phi_{Y} f,
$$

for all $Y \in \mathbf{P}(n)$. Q.E.D. 
3. The infinitesimal intertwining maps. Fix a finite-dimensional irreducible representation $[H, \mu]$ of the subgroup $M(n)$. We will now construct all the intertwining maps from the $U(\mathbf{G}(n))$-module $\left[d C_{\mu}(K(n), H), d \Pi_{\Lambda}\right]$ to the $U(\mathbf{G}(n))$-module $\left[d C_{\bar{\mu}}(K(n), H), d \Pi_{\Lambda^{\prime}}\right]$. Let $T$ denote such a map. The Fourier components of $T$ can then be computed from the recursion relations in Lemma 1. In order to facilitate this computation, we define some auxiliary rational functions, depending on the class $[\mu]$ and some integer parameters.

Let $\Lambda_{\omega}$ be the highest weight of a class $[\omega] \in \Omega(K(n))$, and if $n \geqslant 3$, let $\Lambda_{\mu}$ be the highest weight of a class $[\mu] \in \Omega(M(n))$. We will assume that the class $[\omega] \in \Omega(K(n))$ is $\mu$-admissible. Then it follows from the branching rule that $\Lambda_{\omega}=\Sigma_{1}^{p} \Lambda_{\omega i} \epsilon_{i}$, where the components $\Lambda_{\omega i}$ can be expressed in the following form:

If $n=2 p+1$, then $\Lambda_{\mu}=\Sigma_{1}^{p} \Lambda_{\mu i} \epsilon_{i}$, where the components $\Lambda_{\mu i}$ are all integers or half odd integers which satisfy the inequalities:

$$
\Lambda_{\mu 1} \geqslant \Lambda_{\mu 2} \geqslant \cdots \geqslant\left|\Lambda_{\mu p}\right| \text {. }
$$

Then we may write $\Lambda_{\omega i}=m_{i}+\left|\Lambda_{\mu i}\right|$, for $1 \leqslant i \leqslant p$, where $m_{i}$ is a nonnegative integer which satisfies one of the inequalities:

$$
m_{1} \geqslant 0, \quad 0 \leqslant m_{i} \leqslant\left|\Lambda_{\mu i}\right|-\Lambda_{\mu i-1} \quad(2 \leqslant i \leqslant p) .
$$

If $n=2 p$, with $p \geqslant 2$, then $\Lambda_{\mu}=\Sigma_{1}^{p-1} \Lambda_{\mu i} \epsilon_{i}$ where, for $1 \leqslant i \leqslant p-1$, the components $\Lambda_{\mu i}$ are all integers or half odd integers which satisfy the inequalities:

$$
\Lambda_{\mu 1} \geqslant \Lambda_{\mu 2} \geqslant \cdots \geqslant \Lambda_{\mu p-1} \geqslant 0 .
$$

In this case we may write for the components of $\Lambda_{\omega}, \Lambda_{\omega i}=m_{i}+\Lambda_{\mu i}$ for $1 \leqslant$ $i \leqslant p-1$, and $\Lambda_{\omega p}=m_{p}-\Lambda_{\mu p-1}$, where $m_{i}$ is for each index $i$ a nonnegative integer which satisfies one of the conditions:

Now assume that $n=2 p$, for $p>1$. For each class $[\mu] \in \Omega(M(n))$, index $i, 1 \leqslant i \leqslant p$, and integer $m_{i}$ satisfying (3b), we define a rational function $\tau_{n i}\left(\mu, \cdot, m_{i}\right)$ on $\mathbf{C}$ as follows:

(4) $\tau_{n i}(\mu, \nu, 0)=1, \quad \tau_{n i}\left(\mu, \nu, m_{i}\right)=\prod_{j=0}^{m_{i}-1} \frac{\left(\Lambda_{\mu i}+p-i+1 / 2+j-\nu\right)}{\left(\Lambda_{\mu i}+p-i+1 / 2+j+\nu\right)}$

if $1 \leqslant i \leqslant p-1$ and $m_{i}>0$, and

$$
\tau_{n p}\left(\mu, \nu, m_{p}\right)=\prod_{j=0}^{m_{p}-1} \frac{\left(-\Lambda_{\mu p-1}+1 / 2+j-\nu\right)}{\left(-\Lambda_{\mu p-1}+1 / 2+j+\nu\right)},
$$

if $m_{p}>0$. 
In case $\nu$ is a pole for one of the meromorphic functions defined above, we define additional complex numbers as follows.

For some index $i$ with $1 \leqslant i<p$ assume that there exists a nonnegative integer $n_{i}$ which satisfies condition (3b) and such that $n_{i}+1$ also satisfies condition (3b). Assume also that $\nu$ is given by $\nu=-\left(\Lambda_{\mu i}+p-i+1 / 2+n_{i}\right)$. Then $\nu$ is a pole for all $\tau_{n i}\left(\mu, \cdot, m_{i}\right)$ with $m_{i} \geqslant n_{i}+1$. For this value of $\nu$ we set

$$
\begin{aligned}
\tau_{n i}\left(\mu, \nu, m_{i}\right) & =0, \text { for } 0 \leqslant m_{i} \leqslant n_{i}, \\
\tau_{n i}\left(\mu, \nu, n_{i}+1\right) & =1, \\
\tau_{n i}\left(\mu, \nu, m_{i}\right) & =\prod_{j=n_{i}+1}^{m_{i}-1} \frac{\Lambda_{\mu i}+p-i+1 / 2+j-\nu}{\Lambda_{\mu i}+p-i+1 / 2+j+\nu} \\
& =\left(\begin{array}{c}
2 \Lambda_{\mu i}+n-2 i+m_{i}+n_{i} \\
m_{i}-n_{i}-1
\end{array}\right), \text { if } n_{i}+2 \leqslant m_{i} .
\end{aligned}
$$

Now, suppose $\Lambda_{\mu p-1} \neq 0$, and there exists a positive integer $n_{p}$ such that $0<n_{p} \leqslant 2 \Lambda_{\mu p-1}$, and such that $\nu=n_{p}-\Lambda_{\mu p-1}-1 / 2$. In this case there must also exist a positive integer $n_{p}^{\prime}$ such that $0<n_{p}^{\prime} \leqslant 2 \Lambda_{\mu p-1}$ and such that $\nu=\Lambda_{\mu p-1}+1 / 2-n_{p}^{\prime}$. We must distinguish between two cases:

\section{Case 1. $n_{p} \leqslant n_{p}^{\prime}$.}

Case 2. $n_{p}>n_{p}^{\prime}$.

In Case 1 we have $\nu=\left(n_{p}-n_{p}^{\prime}\right) / 2 \leqslant 0$. We define two functions on the set of integers $m_{p}$ which satisfy the inequality $0 \leqslant m_{p} \leqslant 2 \Lambda_{\mu p-1}$.

$$
\begin{aligned}
\tau_{n p}^{-}(\mu, \nu, 0) & =1, \\
\tau_{n p}^{-}\left(\mu, \nu, m_{p}\right) & =\tau_{n p}\left(\mu, \nu, m_{p}\right) \\
& =\prod_{j=0}^{m_{p}^{-1}} \frac{\left(j+1-n_{p}\right)}{\left.j+1-n_{p}^{\prime}\right)}, \text { for } 0<m_{p}<n_{p}^{\prime}, \\
& =0, \quad \text { for } n_{p}^{\prime} \leqslant m_{p} \leqslant 2 \Lambda_{\mu p-1} .
\end{aligned}
$$

$$
\tau_{n p}^{+}\left(\mu, \nu, m_{p}\right)=0, \text { for } 0 \leqslant m_{p} \leqslant n_{p} \text {, }
$$

$$
=\prod_{j=m_{p}}^{2 \Lambda_{\mu p-1}-1} \frac{\left(j+1-n_{p}^{\prime}\right)}{\left(j+1-n_{p}\right)}, \text { for } n_{p}<m_{p} \leqslant 2 \Lambda_{\mu p-1},
$$

$\tau_{n p}^{+}\left(\mu, \nu, 2 \Lambda_{\mu p-1}\right)=1$.

In Case 2 we have $\nu=\left(n_{p}-n_{p}^{\prime}\right) / 2>0$, and we define one function on the set of integers which satisfy the inequality $0 \leqslant m_{p} \leqslant 2 \Lambda_{\mu p-1}$. 
(8)

$$
\begin{aligned}
\tau_{n p}^{F}\left(\mu, \nu, m_{p}\right) & =0, \text { for } 0 \leqslant m_{p} \leqslant n_{p}^{\prime}-1, \\
\tau_{n p}^{F}\left(\mu, \nu, n_{p}^{\prime}\right) & =1, \\
\tau_{n p}^{F}\left(\mu, \nu, m_{p}\right) & =\prod_{j=n_{p}^{+1}}^{m_{p}} \frac{\left(j-n_{p}\right)}{\left(j-n_{p}^{\prime}\right)}, \text { for } n_{p}^{\prime}<m_{p} \leqslant 2 \Lambda_{\mu p-1} .
\end{aligned}
$$

Now suppose that $n=2$; so that $p=1$. Then for all $[\omega] \in \Omega(K(2))$, $\Lambda_{\omega}=\Lambda_{\omega_{1}} \epsilon_{1}$, where $\Lambda_{\omega 1}$ is either an integer or a half odd integer, depending on whether $\mu$ is the trivial or the nontrivial representation of $M(2)$, respectively. In the latter case, we write $\mu=-$, and $\Lambda_{\omega_{1}}=1 / 2+m$, with $m \in Z$, while in the former case we write $\mu=+$ and $\Lambda_{\omega_{1}}=m \in Z$. For each integer $m$ we define a rational function $\tau_{21}(+, \nu, m)$ on $\mathrm{C}$ as follows.

$$
\begin{aligned}
& \tau_{21}(+, \nu, 0)=1, \\
& \tau_{21}(+, \nu, m)=\prod_{j=0}^{|m|-1} \frac{(j+1 / 2-v)}{(j+1 / 2+\nu)}, \quad \text { for } m \neq 0 .
\end{aligned}
$$

For each $m \in \mathbf{Z}$ we also define a rational function on $\mathbf{C}$ by

$$
\begin{aligned}
& \tau_{21}(-, \nu, 0)=1 \text {, } \\
& \tau_{21}(-, \nu, m)=\prod_{j=0}^{m-1} \frac{(j+1-\nu)}{(j+1+\nu)}, \quad \text { if } m>0 \\
& \tau_{21}(-, \nu, m)=\prod_{j=0}^{-m-1} \frac{(j-\nu)}{(j+\nu)}, \quad \text { if } m<0
\end{aligned}
$$

We now consider the poles of these functions. If $\mu=+$, a zero for $\tau_{21}(+, \nu, m)$ occurs for some $m$ if there exists an integer $n$ such that $\nu=$ $n+1 / 2$. In this case there exists an integer $n^{\prime}$ such that $\nu=-\left(n^{\prime}+1 / 2\right)$. This value of $\nu$ is a pole for some $\tau_{21}(+, \nu, m)$. Then also $n+n^{\prime}+1=0$. We must distinguish between two cases:

Case 1. $-(n+1)=n^{\prime} \geqslant n$. Then $\nu=\left(n-n^{\prime}\right) / 2<0$.

Case 2. $-(n+1)=n^{\prime} \leqslant n-1$. Then $\nu=\left(n-n^{\prime}\right) / 2 \geqslant 1 / 2$.

In Case 1 we define two functions on $\mathbf{Z}$ as follows.

$$
\tau_{12}^{-}(+, \nu, m)=0, \text { if } n+1 \leqslant m,
$$

(11) $\tau_{21}^{-}(+, \nu, n)=1$,

$$
\tau_{21}^{-}(+, \nu, n)=\prod_{j=0}^{n-m-1} \frac{j-2 n}{j+1}=\left(\begin{array}{c}
-n-m-1 \\
n-m
\end{array}\right), \text { for } m \leqslant n-1 ;
$$




$$
\tau_{21}^{+}(+, v, m)=0, \text { if } m \leqslant n^{\prime}=-(n+1),
$$

$$
\tau_{21}^{+}(+, v,-n)=1
$$

$$
\tau_{21}^{+}(+, v, m)=\prod_{j=0}^{n+m-1} \frac{(j-2 n)}{(j+1)}=\left(\begin{array}{c}
m-n-1 \\
n+m
\end{array}\right), \text { for } m \geqslant-n+1 .
$$

In Case 2, we define only one function on $\mathbf{Z}$ :

$$
\begin{aligned}
& \tau_{21}^{F}(+, \nu, m)=0, \text { for } m \geqslant n+1 \text { and } m \leqslant-n-1, \\
& \tau_{21}^{F}(+, \nu,-n)=1
\end{aligned}
$$

$$
\begin{aligned}
& \tau_{21}^{F}(+, \nu, m)=\prod_{j=0}^{n+m-1} \frac{(j-2 n)}{j+1}=(-1)^{m+n}\left(\begin{array}{c}
2 n \\
m+n
\end{array}\right), \\
& \text { for }-n+1 \leqslant m \leqslant n \text {. }
\end{aligned}
$$

Similarly, if $\mu=-$, a zero occurs for some $\tau_{21}(-, \cdot m)$ when there exists an integer $n$ such that $\nu=n+1$. In that case one must also have $\nu=-\left(n^{\prime}+1\right)$ for some integer $n^{\prime}$. We again distinguish between two cases:

Case 1. $-(n+2)=n^{\prime} \geqslant n$. Then $\nu=\left(n-n^{\prime}\right) / 2 \leqslant 0$.

Case 2. $-(n+2)=n^{\prime} \leqslant n-1$. Then $\nu=\left(n-n^{\prime}\right) / 2 \geqslant 1 / 2$.

In Case 1 we define two functions on $Z$, as before.

$$
\tau_{21}^{-}(-, \nu, m)=0, \text { for } m \geqslant n+1 \text {, }
$$

$$
\begin{aligned}
& \tau_{21}^{-}(-, \nu, n)=1, \\
& \tau_{21}^{-}(-, \nu, m)=\prod_{j=0}^{n-m-1} \frac{j-2 n-1}{j+1}=\left(\begin{array}{c}
-n-m-2 \\
n-m
\end{array}\right), \text { for } m \leqslant n-1 . \\
& \quad \tau_{21}^{+}(-, \nu, m)=0, \text { for } m \leqslant n^{\prime}=-(n+2),
\end{aligned}
$$

(15) $\tau_{21}^{+}(-, \nu,-n-1)=1$,

$$
\tau_{21}^{+}(-, \nu, m)=\prod_{j=0}^{m+n-1} \frac{j-2 n}{j+2}=\left(\begin{array}{c}
m-n-1 \\
m+n+1
\end{array}\right), \text { for } m \geqslant-n
$$

In Case 2 we define one function on $\mathbf{Z}$ :

$$
\tau_{21}^{F \cdot}(-, v, m)=0 \text {, if } m \leqslant n^{\prime}=-(n+2) \text { or } m \geqslant n+1 \text {, }
$$

$$
\begin{aligned}
& \tau_{21}^{F}(-, \nu,-n-1)=1, \\
& \tau_{21}^{F}(-, \nu, m)=\prod_{j=0}^{m+n-1} \frac{j-2 n}{j+2}=(-1)^{m+n}\left(\begin{array}{c}
2 n \\
m+n
\end{array}\right), \\
& -n<m \leqslant n .
\end{aligned}
$$


Now, let $n=2 p+1$. Let $i$ be an index with $1 \leqslant i \leqslant p$, and let $m_{i}$ be an integer satisfying condition 3a. Then we define a rational function $\nu \rightarrow$ $\tau_{n i}\left(\mu, \nu, m_{i}\right)$ on $\mathrm{C}$ as follows. (The absolute value sign is needed only in the case when $i=p$.)

$$
\begin{aligned}
\tau_{n i}(\mu, \nu, 0) & =1 \\
\tau_{n i}\left(\mu, \nu, m_{i}\right) & =\prod_{j=0}^{m_{i}-1} \frac{\left|\Lambda_{\mu i}\right|-i+p+1+j-\nu}{\left|\Lambda_{\mu i}\right|-i+p+1+j+\nu} \text { if } m_{i}>0 .
\end{aligned}
$$

Suppose there exists an index $i$ and a nonnegative integer $n_{i}$ which satisfies condition 3a, and such that $\nu=-\left(\left|\Lambda_{\mu i}\right|+1-i+p+n_{i}\right)$. Assume also that $n_{i}+1$ also satisfies condition 3a. Then $\nu$ is a pole for some function $\tau_{n i}\left(\mu, \nu, m_{i}\right)$. For these values of $\nu$ we define the function $\tau_{n i}\left(\mu, \nu, m_{i}\right)$ as follows.

$$
\begin{aligned}
\tau_{n i}\left(\mu, \nu, m_{i}\right) & =0, \text { if } 0 \leqslant m_{i} \leqslant n_{i}, \\
\tau_{n i}\left(\mu, \nu, n_{i}+1\right) & =1, \\
\tau_{n i}\left(\mu, \nu, m_{i}\right) & =\prod_{j=n_{i}+1}^{m_{i}-1} \frac{\left|\Lambda_{\mu i}\right|-i+p+1+j-\nu}{\left|\Lambda_{\mu i}\right|-i+p+1+j+\nu} \\
& =\left(\begin{array}{c}
2 \Lambda_{\mu}+n-2 i+m_{i}+n_{i} \\
m_{i}-n_{i}-1
\end{array}\right),
\end{aligned}
$$

if $m_{i}$ satisfies condition $3 \mathrm{a}$ and $m_{i} \geqslant n_{i}+2$.

We now write down some candidates for intertwining operators from the $U(\mathbf{G}(n))$-module $\left[d C_{\mu}(K(n), H), d \Pi_{\Lambda}\right]$ to $\left[d C_{\bar{\mu}}(K(n), H), d \Pi_{\Lambda^{-1} \mathbf{p}^{2}}\right]$. First, if $n=2 p$ we impose the following condition:

(C) If $n=2 p$ and $n \geqslant 4$, and if $\Lambda_{\mu p-1} \neq 0$, then $\nu \neq-\Lambda_{\mu p-1}+$ $m+1 / 2$ for all integers $m$ such that $0 \leqslant m<2 \Lambda_{\mu p-1}$. If $n=2$, and $\mu=-$, then $\nu \notin Z+1 / 2$. If $n=2$, and if $\mu=+$, then $\nu \notin Z$.

For each $\mu$-admissible class $[\omega]$ we define a linear map $T_{n}(\mu, \nu, \omega)$ as follows:

$$
\begin{aligned}
T_{n}(\mu, \nu, \omega) & =t_{n}\left(\mu, \nu, \Lambda_{\omega}\right) E_{\omega}, \text { if } \mu=\bar{\mu}, \\
& =w_{n}\left(\mu, \nu, \Lambda_{\omega}\right) I_{\omega}, \text { if } \mu \neq \bar{\mu},
\end{aligned}
$$

where the complex numbers $t_{n}\left(\mu, \nu, \Lambda_{\omega}\right)$ and $w_{n}\left(\mu, \nu, \Lambda_{\omega}\right)$ are given by

$$
\begin{aligned}
t_{n}\left(\mu, \nu, \Lambda_{\omega}\right) & =\prod_{i=1}^{p} \tau_{n i}\left(\mu, \nu, m_{i}\right) ; \\
w_{n}\left(\mu, \nu, \Lambda_{\omega}\right) & =\prod_{i=1}^{p}(-1)^{m} \tau_{i_{n i}}\left(\mu, \nu, m_{i}\right)
\end{aligned}
$$

with the integers $m_{i}$ defined in terms of $[\omega]$ at the beginning of this section.

REMARK. For each class $[\omega] \in \Omega_{\mu}(K(n)), \nu \rightarrow t_{n}\left(\mu, \nu, \Lambda_{\omega}\right)$ is a uniquely 
defined complex valued function on $\mathbf{C}$. In fact, if $\nu$ is not a pole for any of the rational functions $\tau_{n i}\left(\mu, v, m_{i}\right)$, then these functions are defined by formulas (4), (5), (9), (10), or (17). On the other hand, if $\nu$ is a pole of one of these functions, then the number $\tau_{n i}\left(\mu, \nu, m_{i}\right)$ is defined by one of the formulas (6) or (18). Condition $\mathrm{C}$ precludes the possibility that $\nu$ is a pole of the rational functions $\tau_{n p}\left(\mu, \nu, m_{p}\right)$, when $n=2 p$.

We may now write the formal sum:

$$
T_{n}(\mu, \nu)=\sum\left\{T_{n}(\mu, \nu, \omega):[\omega] \in \Omega_{\mu}(K(n))\right\} .
$$

Note that, for each element $f \in d C_{\mu}(K(n), H), T_{n}(\mu, \nu) f$ is a finite sum. Hence, $T_{n}(\mu, \nu)$ defines a linear map from $d C_{\mu}(K(n), H)$ to $d C_{\mu}(K(n), H)$.

Now let $n=2 p$. Assume that $\Lambda_{\mu p-1} \neq 0$, if $p>1$, and that condition $\mathrm{C}$ is violated. Then there exists a pair of integers $n_{p}$ and $n_{p}^{\prime}$ such that

$$
\begin{aligned}
\nu & =n_{p}-\Lambda_{\mu p-1}-1 / 2=\Lambda_{\mu p-1}+1 / 2-n_{p}^{\prime} & & \text { if } p>1, \\
& =n_{1}+1 / 2=-n_{1}^{\prime}-1 / 2 & & \text { if } \mu=+ \text { and } p=1, \\
& =n_{1}+1=-n_{1}^{\prime}-1 & & \text { if } \mu=- \text { and } p=1 .
\end{aligned}
$$

We again distinguish two cases:

Case 1. $n_{p}^{\prime} \geqslant n_{p}$. Then $\nu=\left(n_{p}-n_{p}^{\prime}\right) / 2 \leqslant 0$.

Case 2. $n_{p}^{\prime}<n_{p}$. Then $\nu=\left(n_{p}-n_{p}^{\prime}\right) / 2>0$.

In Case 1 we define for each class $[\omega] \in \Omega_{\mu}(K(n))$, two linear operators $T_{n}^{+}(\mu, \nu, \omega)$ and $T_{n}^{-}(\mu, \nu, \omega)$ by the formula

$$
T_{n}^{ \pm}(\mu, \nu, \omega)=t_{n}^{ \pm}(\mu, \nu, \omega) E_{\omega},
$$

where $t_{n}^{ \pm}(\mu, \nu, \omega)$ is defined by the product:

$$
\begin{aligned}
t_{n}^{ \pm}(\mu, \nu, \omega) & =\tau_{n p}^{ \pm}\left(\mu, \nu, m_{p}\right) \prod_{i=1}^{p-1} \tau_{n i}\left(\mu, \nu, m_{i}\right), & & \text { if } p>1, \\
& =\tau_{21}^{ \pm}\left(\mu, \nu, m_{1}\right), & & \text { if } p=1 .
\end{aligned}
$$

Again, we define the formal sums $T_{n}^{ \pm}(\mu, \nu)=\Sigma\left\{T_{n}^{ \pm}(\mu, \nu, \omega):[\mu] \in \Omega_{\mu}(K(n))\right\}$, and note as before that this sum defines a linear map on $d C_{\mu}\left(K(n), f^{\prime}\right)$. In Case 2 we define the linear operator $T_{n}^{F}(\mu, \nu, \omega)$ by

$$
T_{n}^{F}(\mu, \nu, \omega)=t_{n}^{F}(\mu, \nu, \omega) E_{\omega},
$$

where $t_{n}^{F}(\mu, \nu, \omega)$ is given by the product:

$$
\begin{aligned}
t_{n}^{F}(\mu, \nu, \omega) & =\tau_{n p}^{F}\left(\mu, \nu, m_{p}\right) \prod_{i=1}^{p-1} \tau_{n i}^{F}\left(\mu, \nu, m_{i}\right), & & \text { if } p>1, \\
& =\tau_{21}^{F}\left(\mu, \nu, m_{1}\right), & & \text { if } p=1 .
\end{aligned}
$$


Again, we write the formal sum $T_{n}^{F}(\mu, \nu)=\Sigma\left\{T_{n}^{F}(\mu, \nu, \omega):[\omega] \in \Omega_{\mu}(K(n))\right\}$, and point out that this sum defines a linear map on $d C_{\mu}(K(n), H)$.

THEOREM 1. Let $[H, \mu]$ be an irreducible finite-dimensional representation of the subgroup $M(n)$. Let $\Lambda$ be a complex character of the subgroup $S(n)$. We write $\nu=d \Lambda(H)-(n-1) / 2$; so that $-\nu=d \Lambda^{\prime}(H)-(n-1) / 2$, where $\Lambda^{\prime}$ is the Weyl conjugate character $\Lambda^{\prime}=\Lambda^{-1} \mathrm{P}^{2}$.

(1) If condition $\mathrm{C}$ holds, then the space of intertwining maps:

$$
V=\operatorname{HOM}_{U(\mathrm{G}(n))}\left(\left[d C_{\mu}(K(n), H), d \Pi_{\Lambda}\right],\left[d C_{\mu}(K(n), H), d \Pi_{\Lambda^{\prime}}\right]\right)
$$

has complex dimension 1 , and is spanned by the linear map $T_{n}(\mu, \nu)$.

(2) Assume $n=2 p$, and that if $n \geqslant 4, \Lambda_{\mu p-1} \neq 0$. Assume also that condition $\mathrm{C}$ does not hold. Then in Case 1 , the complex dimension of $V$ is equal to 2 , and is given by the span:

$$
V=\operatorname{span}_{\mathbf{C}}\left\{T_{n}^{+}(\mu, \nu), T_{n}^{-}(\mu, \nu)\right\} .
$$

In Case 2 the space of intertwining maps has complex dimension equal to 1 and is given by $V=\mathbf{C} T_{n}^{F}(\mu, v)$.

4. Proof of Theorem 1. We state and prove some simple lemmas which will be needed in the proof of Theorem 1, as well as in the subsequent discussion. Again, $[H, \mu]$ is a finite-dimensional irreducible representation of the subgroup $M(n)$, and $\nu$ is the parameter defined in terms of the character $\Lambda$ in the last section.

Lemma 4. If $n=2 p+1$, let $i$ be an index with $1 \leqslant i \leqslant p$. If $n=2 p$, and $n \geqslant 4$, let $i$ be an index with $1 \leqslant i<p$.

(a) Assume that for all integers $n_{i}$ such that $n_{i}$ and $n_{i}+1$ both satisfy condition $3 \mathrm{a}$ or $3 \mathrm{~b}$, we have $\nu \neq-\left(\Lambda_{\mu i}-i+(n+1) / 2+n_{i}\right)$. (In order for such integers $n_{i}$ to exist, we must have $i=1$, or $\Lambda_{\mu i-1}>\Lambda_{\mu i}$.) Then for any complex number $\alpha$, the sequence:

$$
\left\{z\left(m_{i}\right)=\alpha \tau_{n i}\left(\mu, \nu, m_{i}\right): m_{i} \text { satisfies } 3 \mathrm{a}, \mathrm{b}\right\}
$$

is the unique solution sequence of the difference equations 1a such that $z(0)=\alpha$.

(b) Assume the hypotheses of (a). Assume moreover, that there does exist an integer $n_{i}$ such that both $n_{i}$ and $n_{i}+1$ satisfy the conditions $3 \mathrm{a}, \mathrm{b}$. Then the solution $\tau_{n i}\left(\mu, \nu, m_{i}\right)$ is equal to zero for all $m_{i} \geqslant n_{i}+1$, where $m_{i}$ satisfies 3a, b, and this solution is different from zero for all $0 \leqslant m_{i} \leqslant n_{i}$.

(c) Assume that there exists an integer $n_{i}$ such that both $n_{i}$ and $n_{i}+1$ satisfy conditions $3 \mathrm{a}, \mathrm{b}$, and such that $\nu=-\left(\Lambda_{\mu i}-i+(n+1) / 2+n_{i}\right)$. Then for any complex number $\alpha, Z(\cdot)=\alpha \tau_{n i}(\mu, \nu, \cdot)$ is the unique solution of the 
difference equation 1a such that $Z\left(n_{i}+1\right)=\alpha$. In this case $\tau_{n i}\left(\mu, \nu, m_{i}\right)=0$ for all $m_{i}$ such that $0 \leqslant m_{i} \leqslant n_{i}$.

Proof. If $i>1$, and $\Lambda_{\mu i}=\Lambda_{\mu i-1}$, then 0 is the only integer which satisfies conditions $3 a, b$. Then by the definition in equations (4) and (18), $\alpha \tau_{n i}(\mu, \nu, 0)=\alpha$, and there is nothing to prove.

The equations (1) can be written in the following form, if $m_{i}$ is an integer such that $m_{i}$ and $m_{i}+1$ both satisfy conditions $3 \mathrm{a}, \mathrm{b}$ :

$\left(\Lambda_{\mu i}+m_{i}-i+(n+1) / 2-\nu\right) z\left(m_{i}\right)=\left(\Lambda_{\mu i}+m_{i}-i+(n+1) / 2+\nu\right) z\left(m_{i}+1\right)$.

The condition of statement (a) guarantees that the coefficient on the right-hand side of this equation is never equal to zero. Hence statement (a) follows by a simple induction argument. Statement (b) is obvious from the explicit formula for $\tau_{n i}\left(\mu, \nu, m_{i}\right)$. If the hypothesis of statement (c) holds, then the coefficient of the left-hand side of the above equation is never equal to zero. Hence, the last sentence follows by downward recursion, while the remaining conclusion follows by upward recursion, and substitution of the value of $\nu$. Q.E.D.

Lemma 5. Assume that $n \geqslant 3$. Let $i$ be an index with $1 \leqslant i \leqslant p$. Suppose $n_{i}$ is an integer such that $n_{i}$ and $n_{i}+1$ both satisfy conditions $3 \mathrm{a}, \mathrm{b}$, and such that

$$
\nu= \pm\left(\Lambda_{\mu i}+n_{i}-i+(n+1) / 2\right), \text { or } \nu= \pm\left(\Lambda_{\mu p-1}+n_{p}+1 / 2\right)
$$

in case $n=2 p$ and $i=p$. Then this index is unique.

Proof. The proof of this lemma is contained in the proofs of Theorems 3,4 , and 5 of [10]. Q.E.D.

Lemma 6. Suppose $n=2 p \geqslant 4$. Assume that $\Lambda_{\mu p-1} \neq 0$, and assume that there exists an integer $n_{p}$ such that $0<n_{p} \leqslant 2 \Lambda_{\mu p-1}$, and such that $\nu=n_{p}-\Lambda_{\mu p-1}-1 / 2$. Then there exists an integer $n_{p}^{\prime}$ such that $\nu=\Lambda_{\mu p-1}+$ $1 / 2-n_{p}^{\prime}$. In Case $1, n_{p}^{\prime} \geqslant n_{p}$, and every sequence $\left\{z\left(m_{p}\right): 0 \leqslant m_{p} \leqslant 2 \Lambda_{\mu p-1}\right\}$ which satisfies the difference equation:

$$
\left(-\Lambda_{\mu p-1}+m_{p}+1 / 2-\nu\right) z\left(m_{p}\right)=\left(-\Lambda_{\mu p-1}+m_{p}+1 / 2+\nu\right) z\left(m_{p}+1\right)
$$

lies in the two-dimensional sequence space

$$
\left\{\alpha \tau_{n p}^{+}\left(\mu, \nu, m_{p}\right)+\beta \tau_{n p}^{-}\left(\mu, \nu, m_{p}\right): 0 \leqslant m_{p} \leqslant 2 \Lambda_{\mu p-1}, \alpha, \beta \in \mathbf{C}\right\} .
$$

In Case $2, n_{p}^{\prime}<n_{p}$, and every sequence $\left\{z\left(m_{p}\right): 0 \leqslant m_{p} \leqslant 2 \Lambda_{\mu p-1}\right\}$ which satisfies the above difference equation must be proportional to the sequence $\left\{\tau_{n p}^{F}\left(\mu, \nu, m_{p}\right): 0 \leqslant m_{p} \leqslant 2 \Lambda_{\mu p-1}\right\}$. 
In Case 1, $\tau_{n p}^{-}\left(\mu, \nu, m_{p}\right)=0$ for all integers $m_{p}$ such that $n_{p} \leqslant m_{p} \leqslant$ $2 \Lambda_{\mu p-1}$ and $\tau_{n p}^{+}\left(\mu, \nu, m_{p}\right)=0$ for all $m_{p}$ such that $0 \leqslant m_{p} \leqslant n_{p}^{\prime}$.

In Case 2, $\tau_{n p}^{F}\left(\mu, \nu, m_{p}\right)=0$ for all integers $m_{p}$ such that $0 \leqslant m_{p}<n_{p}^{\prime}$ and $n_{p} \leqslant m_{p} \leqslant 2 \Lambda_{\mu p-1}$.

Finally, suppose $n=2 p \geqslant 2$, and condition $\mathrm{C}$ holds; then for $i=p, \nu \in \mathrm{C}$, the sequence $\left\{z\left(m_{p}\right)\right\}=\left\{\alpha \tau_{n p}\left(\mu, \nu, m_{p}\right)\right\}$ is the unique solution sequence of (1a) with $m_{p}$ satisfying $3 \mathrm{~b}$, and $z(0)=\alpha$.

Proof. The first remark of the lemma was already indicated in the discussion preceding equation (7), and is trivial. Next note that by eliminating the parameter $\nu$, one can write the equation of the lemma in the form:

$$
\left(-n_{p}+1+j\right) z(j)=\left(j+1-n_{p}^{\prime}\right) z(j+1) .
$$

Let $\left\{z(j): 0 \leqslant j \leqslant 2 \Lambda_{\mu p-1}\right\}$ be a solution sequence to this equation. In Case 1 we write $\alpha=z(0)$, and $\beta=z\left(2 \Lambda_{\mu p-1}\right)$. By upward recursion one can solve equation (*) for all integers $m_{p}$ such that $0 \leqslant m_{p}<n_{p}^{\prime}$, since for those integers the coefficient of the right-hand side of $(*)$ is never equal to zero. For those integers we get $z\left(m_{p}\right)=\alpha \tau_{n p}^{-}\left(\mu, \nu, m_{p}\right)$. By downward recursion, one can solve equation (*) for all integers $m_{p}$ such that $n_{p} \leqslant m_{p} \leqslant 2 \Lambda_{\mu p-1}$, since for those integers the left-hand side of $(*)$ is never zero. In this case we have $z\left(m_{p}\right)=$ $\beta \tau_{n p}^{+}\left(\mu, \nu, m_{p}\right)$. Now it is obvious from the explicit formulas in equation (7), that $\tau_{n p}^{+}\left(\mu, \nu, m_{p}\right)=0$ for $m_{p} \leqslant n_{p}^{\prime}$, and that $\tau_{n p}^{-}\left(\mu, \nu, m_{p}\right)=0$ for all $m_{p}$ such that $n_{p} \leqslant m_{p} \leqslant 2 \Lambda_{\mu p-1}$. Hence,

$$
z\left(m_{p}\right)=\alpha \tau_{n p}^{-}\left(\mu, v, m_{p}\right)+\beta \tau_{n p}^{+}\left(\mu, v, m_{p}\right)
$$

for all integers $m_{p}$ such that $0 \leqslant m_{p} \leqslant 2 \Lambda_{\mu p-1}$. We have also proved, by the last remark, the last statement of the lemma concerning the function $\tau_{n p}^{ \pm}(\mu, \nu, \cdot)$.

In Case 2 , let $\alpha=z\left(n_{p}^{\prime}\right)$. Since the right-hand side of equation $(*)$ is equal to zero when $j=n_{p}^{\prime}-1$, it follows by downward recursion, that $z\left(m_{p}\right)=0$ for all integers $m_{p}$ such that $0 \leqslant m_{p} \leqslant n_{p}^{\prime}-1$. On the other hand, $m_{p}-n_{p}^{\prime}+$ $1 \neq 0$ for all $m_{p}$ such that $n_{p}^{\prime}<m_{p} \leqslant 2 \Lambda_{\mu p-1}$. Hence, by upward recursion, we must have $z\left(m_{p}\right)=\alpha \tau_{n p}^{F}\left(\mu, \nu, m_{p}\right)$ for all integers $m_{p}$ such that $n_{p}^{\prime} \leqslant$ $m_{p} \leqslant 2 \Lambda_{\mu p-1}$. Hence, by the definition in equation (8), the last equation is true for all integers $m_{p}$ which satisfy the inequality $0 \leqslant m_{p} \leqslant 2 \Lambda_{\mu p-1}$. The last statement of the lemma concerning the function $\tau_{n p}^{F}(\mu, \nu, \cdot)$ is now obvious from the explicit formula in equation (8).

The proof of the last statement is similar to the proof of statement 1 of Lemma 4. The details are left to the reader. Q.E.D.

The singular cases for $n=2$ are treated in the following lemma. 
Lemma 7. Suppose $\mu=+$ and $\nu \notin \mathbf{Z}$, or $\mu=-$, and $\nu \notin \mathbf{Z}+1 / 2$. Then for any complex number $\alpha$, the sequence $\left\{z(m)=\tau_{21}( \pm, \nu, m): m \in \mathbf{Z}\right\}$ is the unique solution of the recursion relation (1a), with $i=p=1$, and $\Lambda_{\omega_{1}}=m$ if $\mu=+$, or $\Lambda_{\omega 1}=m+1 / 2$ if $\mu=-$, and such that $z(0)=\alpha$.

Suppose that $\nu-1 / 2 \in Z$. If $\mu=+$, set $n^{\prime}=-n-1$. If $\mu=-$, set $n^{\prime}=$ $-n-2$. In Case $1, \nu=\left(n-n^{\prime}\right) / 2 \leqslant 0$. In this case every sequence $\{z(m)$ : $m \in \mathbf{Z}\}$ which satisfies the difference equation

$$
(m+1 / 2-v) z(m)=(m+1 / 2+v) z(m+1)
$$

must be in the two-dimensional sequence space:

$$
\left\{\alpha \tau_{21}^{+}(+, \nu, m)+\beta \tau_{21}^{-}(+, \nu, m): m \in \mathbf{Z}, \alpha, \beta \in \mathbf{C}\right\},
$$

and every sequence $\{z(m): m \in \mathbf{Z}\}$ which satisfies the difference equation

$$
(m+1-\nu) z(m)=(m+1+\nu) z(m+1)
$$

lies in the two-dimensional sequence space:

$$
\left\{\alpha \tau_{21}^{+}(-, \nu, m)+\beta \tau_{21}^{-}(-, \nu, m): m \in \mathbf{Z}, \alpha, \beta \in \mathbf{C}\right\} .
$$

In Case 2 we have $\nu=\left(n-n^{\prime}\right) / 2>0$. In this case, every sequence $\{z(m)\}$ which satisfies equation (19) must be proportional to the sequence $\left\{\tau_{21}^{F}(+, \nu,-n)\right\}$, and every sequence which satisfies equation (20) must be proportional to the sequence $\left\{\tau_{21}^{F}(-, \nu,-n)\right\}$.

Finally, in Case 1 , the factors $\tau_{21}^{+}( \pm, \nu, m), \tau_{21}^{-}( \pm, \nu, m)$ are zero on the following sets of integers $m$ :

$$
\begin{array}{ll}
\tau_{21}^{+}(+, \nu, m)=0 \text { for } m \leqslant-(n+1), & \tau_{21}^{+}(-, \nu, m)=0 \text { for } m \leqslant-(n+2), \\
\tau_{21}^{-}(+, \nu, m)=0 \text { for } m \geqslant(n+1), & \tau_{21}^{-}(-, \nu, m)=0 \text { for } m \geqslant n+1 .
\end{array}
$$

In Case 2 the factors $\tau_{21}^{F}( \pm, \nu, m)$ are zero for the following integers $m$ :

$$
\begin{array}{ll}
\tau_{21}^{F}(+, v, m)=0 & \text { for } m \geqslant n+1 \text { or } m \leqslant-n-1, \\
\tau_{21}^{F}(-, v, m)=0 & \text { for } m \leqslant-(n+2) \text { or } m \geqslant n+1 .
\end{array}
$$

Proof. The proof of this lemma is similar to the proof of Lemma 6, with some obvious simplifications. The details are left to the reader. Q.E.D.

We now turn to the proof of the theorem in the case when $n=2 p$.

The following result is known from Theorem 1 in [10]. Let $Y \in \mathrm{P}(n),[\omega]$, $\left[\omega^{\prime}\right] \in \Omega_{\mu}(K(n))$ and $f \in d C_{\mu}(K(n), H)$. Then $E_{\omega^{\prime}} d \Pi_{\Lambda}(Y) E_{\omega} f=0$, unless $\sigma=\Lambda_{\omega^{\prime}}-\Lambda_{\omega}$ is a weight in the $\mathrm{K}(n)_{\mathrm{C}}$-module $\left[\mathrm{P}(n)_{\mathrm{C}}\right.$, ad]. If $\sigma$ is such a weight, then we have $\sigma= \pm \epsilon_{i}$, with $1 \leqslant i \leqslant p$, and 


$$
\begin{aligned}
& E_{\omega^{\prime}} d \Pi_{\Lambda}(Y) E_{\omega} f=\left[\Lambda_{\omega i}+\nu+(n+1) / 2-i\right] E_{\omega^{\prime}} \Phi_{Y} E_{\omega} f, \text { if } \sigma=\epsilon_{i}, \\
& E_{\omega^{\prime}} d \Pi_{\Lambda}(Y) E_{\omega} f=\left[-\Lambda_{\omega i}+\nu-(n-1) / 2+i\right] E_{\omega^{\prime}} \Phi_{Y} E_{\omega} f, \quad \text { if } \sigma=-\epsilon_{i} .
\end{aligned}
$$

Now suppose condition $C$ is true. Then the operator $T_{n}(\mu, \nu)$ is defined. Assume now that $f$ lies in the subspace $E_{\omega} d C_{\mu}(K(n), H)$. We then have from equations (19) and (21), the following expression for $T_{n}(\mu, \nu) d \Pi_{\Lambda}(Y) f$, with $Y \in \mathbf{P}(n):$

$$
\begin{aligned}
T_{n}(\mu, \nu) d \Pi_{\Lambda}(Y) f \\
=\sum_{i=1}^{p} T_{n}(\mu, \nu) E_{\Lambda_{\omega}+\epsilon_{i}} d \Pi_{\Lambda}(Y) f+\sum_{i=1}^{p} T_{n}(\mu, \nu) E_{\Lambda_{\omega}-\epsilon_{i}} d \Pi_{\Lambda}(Y) f \\
=\sum_{i=1}^{p} t_{n}\left(\mu, \nu, \Lambda_{\omega}+\epsilon_{i}\right)\left(\Lambda_{\omega i}+(n+1) / 2-i+\nu\right) E_{\Lambda_{\omega}+\epsilon_{i}} \Phi_{Y} f \\
\quad+\sum_{i=1}^{p} t_{n}\left(\mu, \nu, \Lambda_{\omega}-\epsilon_{i}\right)\left(\Lambda_{\omega i}-(n-1) / 2+i+\nu\right) E_{\Lambda_{\omega}-\epsilon_{i}} \Phi_{Y} f
\end{aligned}
$$

If $\left[\omega^{\prime}\right]$ is a class in $\Omega(K(n))$ which is not $\mu$-admissible, then by the Frobenius reciprocity theorem, $E_{\omega^{\prime}} d C_{\mu}(K(n), H)=0$. On the other hand, if $\left[\omega^{\prime}\right]=\left[\Lambda_{\omega} \pm \epsilon_{i}\right] \in \Omega_{\mu}(K(n))$, then by equation (20) and the last statement of Lemma 6 , if $i=p$, or Lemma 4 if $i<p$, we have

$$
\begin{aligned}
& \left(\nu+(n+1) / 2-i+\Lambda_{\omega i}\right) t_{n}\left(\mu, \nu, \Lambda_{\omega}+\epsilon_{i}\right)=\left(\Lambda_{\omega i}-i+(n+1) / 2-\nu\right) t_{n}\left(\mu, \nu, \Lambda_{\omega}\right), \\
& \left(\nu-(n+1) / 2+i-\Lambda_{\omega i}\right) t_{n}\left(\mu, \nu, \Lambda_{\omega}-\epsilon_{i}\right)=\left(-\Lambda_{\omega i}+i+(n-1) / 2-\nu\right) t_{n}\left(\mu, \nu, \Lambda_{\omega}\right) .
\end{aligned}
$$

Hence for all $Y \in \mathbf{P}(n)$, and $f \in E_{\omega} L_{\mu}^{2}(K(n), H)$,

$$
\begin{aligned}
T_{n}(\mu, \nu) d \Pi_{\Lambda}(Y) f=t_{n}\left(\mu, \nu, \Lambda_{\omega}\right) \sum_{i=1}^{p}( & E_{\Lambda_{\omega}+\epsilon_{i}}\left(\Lambda_{\omega i}-i+(n+1) / 2-\nu\right) \Phi_{Y} f \\
& \left.+E_{\Lambda_{\omega}-\epsilon_{i}}\left(-\Lambda_{\omega i}+i-(n-1) / 2-\nu\right) \Phi_{Y} f\right) .
\end{aligned}
$$

By equation (28) and the definition of the Fourier component $T_{n}(\mu, \nu, \omega)$,

$$
T_{n}(\mu, \nu) d \Pi_{\Lambda}(Y) f=d \Pi_{\Lambda^{-1} \mathrm{P}^{2}}(Y) T_{n}(\mu, \nu) f .
$$

Hence $T_{n}(\mu, \nu)$ is an intertwining operator, that is, a $U(\mathbf{G}(n))$-module homomorphism. The statement concerning the dimension of the space of homomorphisms follows immediately from the dimensionality of the sequence of factors $\tau_{n i}\left(\mu, \nu, m_{i}\right)$ as stated in Lemmas 4 and 6.

Now suppose $n=2 p$, and that condition $\mathrm{C}$ is violated. In Case 1 the two operators $T_{n}^{ \pm}(\mu, \nu)$ are defined. An argument formally identical to the above 
argument shows that these operators have the intertwining property. If $1 \leqslant i<p$, then the sequence of factors $\left\{\tau_{n i}\left(\mu, \nu, m_{i}\right)\right\}$ is unique up to a constant, by Lemma 4 , while by Lemma 6 , the sequence spaces spanned by the factor sequences: $\left\{\tau_{n p}\left(\mu, \nu, m_{p}\right)\right\}$ has dimension 2. (In case $n=2$, Lemma 6 in the last statement must be replaced by Lemma 7.) It follows that for each $\mu$-admissible class $[\omega]$, the space spanned by $T_{n}(\mu, \nu, \omega)$ has dimension 2 . Hence, the space spanned by the operators $T_{n}(\mu, \nu)$ also has dimension 2 .

In Case 2, the operator $T_{n}^{F}(\mu, \nu)$ is defined. An obvious modification of the above argument shows that this operator is unique up to a complex constant multiple. Hence the theorem follows for the groups with even $n$.

Now we turn to the case with $n=2 p+1$. In this case, $\sigma=0$ is also a weight in the $\mathbf{K}(n)_{\mathbf{C}}$-module $\left[\mathrm{P}(n)_{\mathbf{C}}\right.$, ad], in addition to the weights $\sigma=\epsilon_{i}$, with $1 \leqslant i \leqslant p$. Then by Theorem 1 of [10], there is in addition to the pair of equations (28), also the equation

$$
E_{\omega} d \Pi_{\Lambda}(Y) E_{\omega} f=\nu E_{\omega} \Phi_{Y} E_{\omega} f
$$

for $f \in d C_{\mu}(K(n), h)$, and $Y \in \mathbf{P}(n)$.

Again, let $f \in E_{\omega} d C_{\mu}(K(n), H)$, and let $Y \in \mathrm{P}(n)$. First assume that $\mu=$ $\bar{\mu}$. Then by Lemma 3, and by equation (28a) we have $E_{\omega} d \Pi_{\Lambda}(Y) E_{\omega}=0$. As before, we compute $T_{n}(\mu, \nu) d \Pi_{\Lambda}(Y) f$, and obtain a sequence of equations identical to (29). Hence the intertwining property of $T_{n}(\mu, \nu)$ follows as before.

Next, assume $\mu \neq \bar{\mu}$. Then we have by the second formulas in (19) and (20),

$$
\begin{aligned}
T_{n}(\mu, \nu) d \Pi_{\Lambda}(Y) f= & \sum_{i=1}^{p} w_{n}\left(\mu, \nu, \Lambda_{\omega}+\epsilon_{i}\right)\left(\Lambda_{\omega i}+(n+1) / 2-i+\nu\right) E_{\omega+\epsilon_{i}} I \Phi_{Y} f \\
& +w_{n}\left(\mu, \nu, \Lambda_{\omega}-\epsilon_{i}\right)\left(\Lambda_{\omega i}-(n-1) / 2+i+\nu\right) E_{\Lambda_{\omega}-\epsilon_{i} I \Phi_{Y} f} \\
& +w_{n}(\mu, \nu, \omega) E_{\omega} I \Phi_{Y} f .
\end{aligned}
$$

Now apply Lemmas 2 and 4 , as before. It follows that $w_{n}\left(\mu, \nu, \Lambda_{\omega}\right)$ and $w_{n}\left(\mu, \nu, \Lambda_{\omega}+\epsilon_{i}\right)$ satisfy equation (1b). We also use, in particular, statement 1 of Lemma 2, and obtain:

$$
\begin{aligned}
& T_{n}(\mu, \nu) d \Pi_{\Lambda}(Y) f \\
& =w_{n}\left(\mu, \nu, \Lambda_{\omega}\right)\left(\sum_{i=1}^{p} E_{\Lambda_{\omega}+\epsilon_{i}}\left(\Lambda_{\omega i}-i+(n+1) / 2-\nu\right) \Phi_{Y} I f\right. \\
& \\
& \left.\quad+\sum_{i=1}^{p} E_{\Lambda_{\omega}-\epsilon_{i}}\left(-\Lambda_{\omega i}+i-(n-1) / 2-\nu\right) \Phi_{Y} I f-E_{\omega} \nu \Phi_{Y} I f\right) \\
& =d \Pi_{\Lambda^{\prime}}(Y) T_{n}(\mu, \nu) f,
\end{aligned}
$$


where again we apply equations (28) and (28a).

The theorem now follows in this case also.

5. Subrepresentations and subquotients. In this section we point out how the results of [10] can be interpreted in terms of the kernels and images of the intertwining maps constructed here. One by-product of this analysis is the result that every subquotient $U(\mathbf{G}(n))$-module in the induced representation modules considered here is equivalent to a submodule of an induced representation module.

As before, let $[H, \mu]$ be a fixed finite-dimensional irreducible representation of the subgroup $M(n)$. Let $\Lambda$ denote a complex character of the subgroup $S(n)$, and let $\Lambda^{\prime}$ denote the Weyl reflected character $\lambda^{\prime}=d \Lambda^{\prime}(H)=-d \Lambda(H)+n-$ $1=-\lambda+n-1$. The parameter $\nu$ is defined in terms of $\lambda$ by the formula $\nu=\lambda-(n-1) / 2$. Expressed in terms of the parameter $\nu$, a necessary and sufficient condition for the irreducibility of the $U(\mathrm{G}(n))$-module $\left[d C_{\mu}(K(n), H)\right.$, $\left.d \Pi_{\Lambda}\right]$ is the following one (see [10, Theorem 6]):

(D) If $i$ is an index such that $1 \leqslant i \leqslant p$, then $\nu+\Lambda_{\omega i}+(n+1) / 2-$ $i \neq 0$, for all $[\omega]^{\cdot} \in \Omega_{\mu}(K(n))$ such that $\left[\Lambda_{\omega}+\epsilon_{i}\right]$ is also in $\Omega_{\mu}(K(n))$, and $\nu-\Lambda_{\omega i}-(n-1) / 2+i \neq 0$ for all $[\omega] \in \Omega_{\mu}(K(n))$ such that $\left[\Lambda_{\omega}-\epsilon_{i}\right]$ is also in $\Omega_{\mu}(K(n))$.

We remark that this condition implies the condition $C$ stated in $\S 3$. The following theorem "explains" this irreducibility criterion in terms of intertwining homomorphisms.

TheOREM 2. (1) Assume that $n \geqslant 3$, and that $i$ is an integer index with $1 \leqslant i \leqslant p$. Assume also, that if $n=2 p$, then $i \neq p$. Assume that there exists a $\mu$-admissible class $\left[\omega^{\prime}\right] \in \Omega_{\mu}(K(n))$ such that $0=\lambda-\Lambda_{\omega^{\prime} i}-n+i$, and such that $\left[\Lambda_{\omega^{\prime}}+\epsilon_{i}\right]$ is also a $\mu$-admissible class. Let $s=\Lambda_{\omega^{\prime} i}$. Then, with $\nu=\lambda-(n-1) / 2$, we have $\operatorname{ker}\left(T_{n}(\mu, \nu)\right)=D_{\lambda}^{+} d C_{\mu}(K(n), H)$, where $D_{\lambda}^{+}$is the projection defined as in [10] by the sum:

$$
D_{\lambda}^{+}=\sum\left\{E_{\omega}:[\omega] \in \Omega_{\mu}(K(n)), \Lambda_{\omega i}>s\right\} .
$$

We recall from [10] the definition of the projection $D_{\lambda^{\prime}}^{-}$by

$$
D_{\lambda^{\prime}}^{-}=\sum\left\{E_{\omega}:[\omega] \in \Omega_{\mu}(K(n)), \Lambda_{\omega i} \leqslant s\right\} .
$$

Then we have $D_{\lambda^{\prime}}^{-}=1-D_{\lambda^{\circ}}^{+}$Then also,

$$
\begin{aligned}
\operatorname{range}\left(T_{n}(\mu, \nu)\right) & =D_{\lambda^{\prime}}^{-} d C_{\bar{\mu}}(K(n), H), \\
\operatorname{ker}\left(T_{n}(\mu,-\nu)\right) & =D_{\lambda^{\prime}}^{-} d C_{\mu}(K(n), H), \\
\operatorname{range}\left(T_{n}(\mu,-\nu)\right) & =D_{\lambda}^{+} d C_{\bar{\mu}}(K(n), H) .
\end{aligned}
$$


(2) Now assume that $n=2 p$. Assume also that there exists a $\mu$-admissible class $\left[\omega^{\prime}\right] \in \Omega_{\mu}(K(n))$ such that $\left[\Lambda_{\omega^{\prime}}+\epsilon_{p}\right]$ is also $\mu$-admissible, and such that $\lambda+\Lambda_{\omega^{\prime} p}-p+1=0$, or equivalently, $\nu+\Lambda_{\omega^{\prime} p}+1 / 2=0$. Set $s=$ $\Lambda_{\omega^{\prime} p}$. We distinguish two cases:

Case 1': $-s>s$. Then $\nu=-s-1 / 2 \geqslant 0$.

Case $2^{\prime}:-s \leqslant s$. Then $\nu=-s-1 / 2<0$.

As in Theorem 5 of [10] we define the following projections:

$$
\begin{aligned}
& D_{\lambda p}^{+}=\sum\left\{E_{\omega}:[\omega] \in \Omega_{\mu}(K(n)), \Lambda_{\omega p} \geqslant-s\right\}, \\
& D_{\lambda p}^{-}=\sum\left\{E_{\omega}:[\omega] \in \Omega_{\mu}(K(n)), \Lambda_{\omega p} \leqslant s\right\},
\end{aligned}
$$

$D_{\lambda^{\prime} p}^{+}=1-D_{\lambda p}^{-}$, and $D_{\lambda^{\prime} p}^{-}=1-D_{\lambda p}^{+}$. In addition, in Case $1^{\prime}$ we define as in [10], $D_{\lambda^{\prime} p}^{F}=D_{\lambda^{\prime} p}^{-} D_{\lambda^{\prime} p}^{+}=D_{\lambda^{\prime} p}^{+} D_{\lambda^{\prime} p}^{-}$, and in Case $2^{\prime}, D_{\lambda p}^{F}=D_{\lambda p}^{-} D_{\lambda p}^{+}=D_{\lambda p}^{+} D_{\lambda p}^{-}$. Then in Case $1^{\prime}$ the linear map $T_{n}^{ \pm}(\mu,-\nu)$ is defined, and we have:

$$
\begin{aligned}
\operatorname{ker}\left(T_{n}^{+}(\mu,-\nu)\right) & =D_{\lambda^{\prime} p}^{-} d C_{\mu}(K(n), H), \\
\operatorname{range}\left(T_{n}^{+}(\mu,-\nu)\right) & =D_{\lambda p}^{+} d C_{\mu}(K(n), H), \\
\operatorname{ker}\left(T_{n}^{-}(\mu,-\nu)\right) & =D_{\lambda^{\prime} p}^{+} d C_{\mu}(K(n), H), \\
\operatorname{range}\left(T_{n}^{-}(\mu,-\nu)\right) & =D_{\lambda p}^{-} d C_{\mu}(K(n), H) .
\end{aligned}
$$

In Case $2^{\prime}$ the linear map $T_{n}^{F}(\mu,-\nu)$ is defined, and we have:

$$
\begin{aligned}
\operatorname{ker}\left(T_{n}^{F}(\mu,-\nu)\right) & =\left(D_{\lambda^{\prime} p}^{+}+D_{\lambda^{\prime} p}^{-}\right) d C_{\mu}(K(n), H), \\
\operatorname{range}\left(T_{n}^{F}(\mu,-\nu)\right) & =D_{\lambda p}^{F} d C_{\mu}(K(n), H) .
\end{aligned}
$$

(3) The map $T_{n}(\mu, \nu)$ is a linear isomorphism from $d C_{\mu}(K(n), H)$ onto $d C_{\bar{\mu}}(K(n), H)$ if and only if condition $\mathrm{D}$ holds.

REMARKS. Under the Weyl reflection: $\Lambda \rightarrow \Lambda^{\prime}=\Lambda^{-1} \mathrm{P}^{2}$ the equation $\lambda+s-p+1$ goes over into the equation $\lambda^{\prime}+s^{\prime}-p+1=0$, where $s^{\prime}$ is given by $s^{\prime}=-s-1$. Hence the equalities between the projections: $D_{\lambda p}^{ \pm}=1-$ $D_{\lambda^{\prime} p}^{\mp}$ follow from the definitions of the projections $D_{\lambda p}^{ \pm}$by replacing $\lambda$ by $\lambda^{\prime}$ and $s$ by $s^{\prime}$ in those definitions. A similar remark applies to the projections defined in statement 1 .

Next we remark that in case $p \geqslant 2$, the condition in statement 2 implies that the component $\Lambda_{\mu p-1}$ is positive.

Finally, we remark that the above theorem may be deduced from Theorem 6 of [10]. However, we shall now show that this theorem follows easily from the results established in the last section.

Proof of the Theorem. First, we note that if there does exist an index 
$i$, with $1 \leqslant i \leqslant p$, and such that condition $\mathrm{D}$ is violated, then by Lemma 5 this index is unique. Assume now that the condition of statement 1 holds; so that we have $\nu=s+(n+1) / 2-i$ for a unique index $i$. Write $s=\Lambda_{\mu i}+n_{i}$. Then $n_{i}$ is a nonnegative integer, and by the condition of statement 1 , both $n_{i}$ and $n_{i}+1$ satisfy conditions $3 \mathrm{a}, \mathrm{b}$. From the definition of the functions $\tau_{n i}\left(\mu, \nu, m_{i}\right)$ in equations (4) or (17), it follows that $\tau_{n i}\left(\mu, \nu, m_{i}\right)=0$ for precisely those integers $m_{i}$ which satisfy the conditions $3 \mathrm{a}, \mathrm{b}$ and the condition $m_{i} \geqslant n_{i}+1$. Moreover, the factors $\tau_{n j}\left(\mu, \nu, m_{j}\right)$ which occur in the definition of $T_{n}(\mu, \nu)$, are different from zero when $j \neq i$. The last fact follows from the explicit formulas for these factors and from the uniqueness of the index $i$. Hence the kernel of $T_{n}(\mu, \nu)$ is spanned by those subspaces $E_{\omega} d C_{\mu}(K(n), H)$ such that $\Lambda_{\omega i} \geqslant$ $n_{i}+1+\Lambda_{\mu i}$. Since such classes exist, this kernel is nontrivial. Since the map $T_{n}(\mu, \nu)$ commutes with the projections $E_{\omega}$, the range of this map is spanned by the complementary set of subspaces: $\left\{E_{\omega} d C_{\mu}(K(n), H):[\omega] \in \Omega_{\mu}(K(n))\right.$, $\left.\Lambda_{\omega i} \leqslant s\right\}$. Hence, the first conclusion of the statement follows.

By the last remark of statement $\mathrm{c}$ in Lemma 4 , the factor $\tau_{n i}\left(\mu,-\nu, m_{i}\right)$ is equal to zero for precisely those $m_{i}$ which satisfy $3 \mathrm{a}, \mathrm{b}$ and the condition $m_{i} \leqslant$ $n_{i}$. Hence by reasoning in a manner similar to the above argument, we have

$$
\operatorname{ker}\left(T_{n}(\mu,-\nu)\right)=\operatorname{span}\left\{E_{\omega} d C_{\mu}(K(n), H): \Lambda_{\omega i} \leqslant n_{i}+\Lambda_{\mu i}\right\}=D_{\lambda^{\prime}}^{-} d C_{\mu}(K(n), H),
$$

and this kernel is nontrivial. The statement concerning the range of the map $T_{n}(\mu,-\nu)$ follows in a similar manner as the statement concerning the range of the map $T_{n}(\mu, \nu)$. Hence, statement 1 is proved.

Under the assumptions of statement 2 of the theorem, condition $C$ of $\S 3$ is violated. Hence, condition $\mathrm{D}$ is violated for the unique index $i=p$. First, let us assume that $p \geqslant 2$. Then $\tau_{n j}\left(\mu, \nu^{\prime}, m_{j}\right) \neq 0$ for all indices $j$ such that $1 \leqslant$ $j<p$, and integers $m_{j}$ which satisfy conditions $3 b$. Hence the kernel of the map $T_{n}(\mu,-\nu)$ may be read off from Lemma 6 . First assume Case $1^{\prime}$ of statement 2. This case corresponds to Case 1 of Lemma 6 with the parameter in that lemma replaced by the parameter $\nu^{\prime}=-\nu$. Write $\lambda^{\prime}=n_{p}-\Lambda_{\mu p-1}-1 / 2=s+1 / 2$. Thus, in terms of $n_{p}$ and $n_{p}^{\prime}$ of Lemma 6 , we have $s=-\Lambda_{\mu p-1}+n_{p}-1$, and $-s=-\Lambda_{\mu p-1}+n_{p}^{\prime}$. Then the factor $\tau_{n p}^{ \pm}\left(\mu, \nu^{\prime}, m_{p}\right)$ is defined for all integers $m_{p}$ which satisfy the inequality: $0 \leqslant m_{p} \leqslant 2 \Lambda_{\mu p-1}$. If $n \geqslant 4$, it follows from Lemma 6 that $\tau_{n p}^{+}\left(\mu, \nu^{\prime}, m_{p}\right)=0$ if and only if $-\Lambda_{\mu p-1} \leqslant-\Lambda_{\mu p-1}+$ $m_{p} \leqslant-s-1$. Hence, the map $T_{n}\left(\mu, \nu^{\prime}\right)$ is defined, and its kernel is correctly indicated in the conclusion of statement 2. It also follows from Lemma 6 that $\tau_{n p}^{-}\left(\mu, \nu^{\prime}, m_{p}\right)=0$, if and only if $s+1=-\Lambda_{\mu p-1}+n_{p} \leqslant-\Lambda_{\mu p-1}+m_{p} \leqslant$ $\Lambda_{\mu p-1}$. It follows that the kernel of the map $T_{n}^{-}\left(\mu, \nu^{\prime}\right)$ is the range of the projection $D_{\lambda^{\prime} p}^{+}$. The statements concerning the ranges of the maps $T_{n}^{ \pm}(\mu,-v)$ again 
follow from the fact that these maps commute with the projections $E_{\omega},[\omega] \in$ $\Omega_{\mu}(K(n))$, as before.

Assume Case $2^{\prime}$ holds. This case corresponds to the Case 2 of Lemma 6, when the parameter $\nu$ in that lemma is replaced by the parameter $\nu^{\prime}=-\nu$. Hence the factor $\tau_{n p}^{F}\left(\mu,-\nu, m_{p}\right)$ is defined for all $m_{p}$ which satisfy the inequalities in $3 b$. Hence the map $T_{n}^{F}(\mu,-\nu)$ is defined. By arguing as above, it follows from Lemma 6 that the kernel of this map consists of the span of $E_{\omega} d C_{\mu}(K(n), H)$ with $-\Lambda_{\mu p-1} \leqslant \Lambda_{\omega p} \leqslant-s-1$ or with $s+1 \leqslant \Lambda_{\omega p} \leqslant$ $\Lambda_{\omega p-1}$. Hence, the kernel of $T_{n}^{F}\left(\mu, \nu^{\prime}\right)$ consists of the subspace

$$
\left(D_{\lambda^{\prime} p}^{-}+D_{\lambda^{\prime} p}^{+}\right) d C_{\mu}(K(n), H) \text {. }
$$

The statement concerning the range of the map $T_{n}^{F}\left(\mu, \nu^{\prime}\right)$ follows from an argument similar to one used above.

In case $n=2$, and $p=1$, statement 2 follows from Lemma 7, by modifying the above argument in an obvious manner.

By the proof of statement 1 the map $T_{n}(\mu, \nu)$ has a nontrivial kernel, if and only if statement $\mathrm{D}$ is false. Hence, if statement $\mathrm{D}$ is true, the map $T_{n}(\mu, \nu)$ exists and is one to one. Moreover, since this map is onto each of the subspaces $E_{\omega} d C_{\bar{\mu}}(K(n), !)$, in this case, $T_{n}(\mu, \nu)$ maps $d C_{\mu}(K(n), H)$ onto $d C_{\bar{\mu}}(K(n), H)$. Q.E.D.

The following facts are known from [10, Theorem 6].

If the assumptions of statement 1 of the last theorem hold, then the $U(\mathbf{G}(n))$-modules $\left[D_{\lambda}^{+} d C_{\mu}(K(n), H), d \Pi_{\Lambda}\right]$ and $\left[D_{\lambda^{\prime}}^{-} d C_{\mu}(K(n), H), d \Pi_{\Lambda^{\prime}}\right]$ are proper irreducible submodules of the $U(\mathbf{G}(n))$-modules $\left[d C_{\mu}(K(n), H), d \Pi_{\Lambda}\right]$, and $\left[d C_{\mu}(K(n), H), d \Pi_{\Lambda^{\prime}}\right]$ respectively. If the assumptions of statement 2 in the above theorem hold, then $\left[D_{\lambda p}^{ \pm} d C_{\mu}(K(n), H), d \Pi_{\Lambda}\right]$ are both $U(\mathrm{G}(n))$-submodules. These submodules are both irreducible if condition $1^{\prime}$ holds. If Case $2^{\prime}$ applies, then the submodule $\left[D_{\lambda p}^{F} d C_{\mu}(K(n), H), d \Pi_{\Lambda}\right]$ is irreducible. Similarly, under the assumptions of statement 2 , the subspaces $D_{\lambda^{\prime} p^{\prime}}^{ \pm} d C_{\mu}(K(n), H)$ are submodules under the action $d \Pi_{\Lambda^{\prime}}$, and are irreducible in Case $2^{\prime}$. In Case $1^{\prime}$, $\left[D_{\lambda^{\prime}{ }^{\prime}}^{F} d C_{\mu}(K(n), H), d \Pi_{\Lambda^{\prime}}\right]$ is irreducible. Finally, if condition $\mathrm{D}$ holds, then the $U(\mathbf{G}(n))$-modules $\left[d C_{\mu}\left(K(n), H^{\prime}\right), d \Pi_{\Lambda}\right]$ and $\left[d C_{\mu}(K(n), H), d \Pi_{\Lambda^{\prime}}\right]$ are both irreducible.

Now, let $T$ be an intertwining map from the $U(\mathbf{G}(n))$-module $\left[d C_{\mu}(K(n), \mathrm{H})\right.$, $\left.d \Pi_{\Lambda}\right]$ into the $U(\mathbf{G}(n))$-module $\left[d C_{\bar{\mu}}\left(K(n), H^{\prime}\right), d \Pi_{\Lambda^{\prime}}\right]$. From a standard theorem on homomorphisms of modules, we may identify the quotient module $\left[d C_{\mu}(K(n), H), d \Pi_{\Lambda}\right] /\left[\operatorname{ker}(T), d \Pi_{\Lambda}\right]$ with the submodule of $\left[d C_{\bar{\mu}}(K(n), H)\right.$, $\left.d \Pi_{\Lambda^{\prime}}\right]$ given by range $(T)$. Hence, by combining the above results with Theorem 2, we obtain with a slight abuse of terminology, the following result.

COROLlary 1. If condition D holds, then $T_{n}(\mu, \nu)$ is an isomorphism 
from the $U(\mathbf{G}(n))$-module $\left[d C_{\mu}(K(n), H), d \Pi_{\Lambda}\right]$ onto the $U(\mathbf{G}(n))$-module $\left[d C_{\bar{\mu}}(K(n), H), d \Pi_{\Lambda^{\prime}}\right]$.

If the assumptions of statement 1 hold, then $T_{n}(\mu, \nu)$ is a $U(\mathrm{G}(n))$-module isomorphism from the quotient module

$$
\left[d C_{\mu}(K(n), H), d \Pi_{\Lambda}\right] /\left[D_{\lambda}^{+} d C_{\mu}(K(n), H), d \Pi_{\Lambda}\right]
$$

onto the submodule $\left[D_{\lambda}^{-} d C_{\bar{\mu}}(K(n), H), d \Pi_{\Lambda^{\prime}}\right]$. Under the same assumptions, $T_{n}(\mu,-\nu)$ is a $U(\mathrm{G}(n))$-module isomorphism from the quotient module

$$
\left[d C_{\mu}(K(n), H), d \Pi_{\Lambda^{\prime}}\right] /\left[D_{\lambda^{\prime}}^{-} d C_{\mu}(K(n), H), d \Pi_{\Lambda^{\prime}}\right]
$$

onto the submodule $\left[D_{\lambda}^{+} d C_{\bar{\mu}}(K(n), H), d \Pi_{\Lambda}\right]$.

As in [10], the subquotient theorem of Harish-Chandra [3b], Theorem 4, leads immediately to the following corollary.

COROLLARY 2. Every irreducible quasi-simple representation of $G(n)$ is infinitesimally equivalent to a subrepresentation of a nonunitary principal series representation.

6. Infinitesimally pseudo-unitary representations. A preliminary step in the classification of the irreducible unitary representations is the study of the infinitesimally pseudo-unitary $U(\mathbf{G}(n))$-representations. This study, which is undertaken in this section, involves some remarks on linear algebra.

We continue the notation of the last section. Let $\left[H, d \Pi_{\Lambda}\right]$ be a nonzero $U(\mathbf{G}(n))$-submodule of the $U(\mathbf{G}(n))$-module $\left[d C_{\mu}(K(n), H), d \Pi_{\Lambda}\right]$. Let $A$ be an Hermitian (not necessarily positive definite) bilinear form on $H$; so that $A(x, y)=\overline{A(y, x)}$, for all $x$ and $y \in H$. The $U(\mathbf{G}(n))$-module $\left[H, d \Pi_{\Lambda}\right]$ will be called infinitesimally pseudo-unitary with respect to the Hermitian form $A$, or if $A$ is understood, infinitesimally pseudo-unitary, provided the following relation holds for all $Y \in \mathrm{G}(n)$, and $x, y \in H$ :

$$
A\left(d \Pi_{\Lambda}(Y) x, y\right)=-A\left(x, d \Pi_{\Lambda}(Y) y\right) .
$$

In other words, for each $Y \in \mathrm{G}(n)$, the linear transformation $d \Pi_{\Lambda}(Y)$ is skewHermitian with respect to the form $A$.

Following Harish-Chandra, we call the $U(\mathbf{G}(n))$-module infinitesimally unitary, if it is infinitesimally pseudo-unitary with respect to some positive definite Hermitian form.

As in [10], we let $\langle$,$\rangle denote the standard inner product on L_{\mu}^{2}(K(n), H)$ defined by means of the Haar integral

$$
\langle f, g\rangle=\int_{K(n)}(f(k), g(k)) d k,
$$

for all $f$, and $g \in L_{\mu}^{2}(K(n), H)$, where $($,$) is an inner product on H$ with 
respect to which $[H, \mu]$ is a unitary representation of $M(n)$.

Note that the restriction of the form $\langle$,$\rangle to the linear subspace H$ is nondegenerate since $\langle$,$\rangle is positive definite.$

Finally, as in [10], we denote complex conjugations on $L_{\mu}^{2}(K(n), H)$ by $\phi \rightarrow \bar{\phi}$.

Let $H^{*}$ denote the linear dual space of $H$, and let $H_{F}$ denote the subset of $H^{*}$ defined by $H_{F}=\left\{f \in H^{*}: f\left(E_{\omega} H\right)=0\right.$ for all but a finite number of classes $\left.[\omega] \in \Omega_{\mu}(K(n))\right\}$. It is clear that $H_{F}$ is a linear subspace of $H^{*}$. The next lemma will justify calling this subspace the subspace of $K(n)$-finite vectors in $H^{*}$. If $f \in H^{*}$, let $\bar{f}$ denote the conjugate linear functional defined by $\bar{f}(x)=\overline{f(x)}$. Then let $\bar{H}_{F}$ denote the linear space of conjugate linear functionals defined by the expression: $\bar{H}_{F}=\left\{\bar{f}: f \in H_{F}\right\}$.

LEMma 8. If $f \in H_{F}$, then there exists an element $x_{f} \in H$ such that $f(y)=\left\langle y, x_{f}\right\rangle$ for all $y \in H$. If $f \in \bar{H}_{F}$, then there exists an element $x_{f} \in H$ such that $f(y)=\left\langle x_{f}, y\right\rangle$, for all $y \in H$. The map $f \rightarrow x_{f}, f \in H_{F}$, is a conjugate linear isomorphism from $H_{F}$ onto $H$. The map $f \rightarrow x_{f}, f \in \bar{H}_{F}$ is a linear isomorphism from $\bar{H}_{F}$ onto $H$.

Proof. Let $f \in H_{F}$, and let $\mathfrak{B}_{f}$ denote the linear span:

$$
\mathfrak{B}_{f}=\operatorname{span}\left\{E_{\omega} H: f\left(E_{\omega} H\right) \neq 0,[\omega] \in \Omega_{\mu}(K(n))\right\} .
$$

Then $\mathfrak{B}_{f}$ has finite dimension and is a Hilbert space under the restriction of the Hermitian form 〈, 〉. Consequently, by the standard Riesz representation theorem, there exists an element $x_{f} \in \mathfrak{B}_{f} \subset H$, such that $f(y)=\left\langle y, x_{f}\right\rangle$ for all $y \in \mathfrak{B}_{f}$. However, for all $y \in \mathfrak{B}_{f} \subset H$ we have $f(y)=0=\left\langle y, x_{f}\right\rangle$. Hence $f(y)=\left\langle y, x_{f}\right\rangle$ for all $y \in H$. The fact that the map $f \rightarrow x_{f}$ is conjugate linear and one to one follows from a standard calculation. The fact that this map is onto follows in a standard way from the nondegeneracy of the Hermitian form 〈, 〉.

The second half of the lemma follows by an analogous argument. Q.E.D.

Corollary 3. The spaces $H, H_{F}$, and $\bar{H}_{F}$ are all linearly isomorphic.

Lemma 9. Let $A$ be an Hermitian form on $H$. Then there exists a linear map $T: H \rightarrow H$ such that $A(x, y)=\langle T x, y\rangle$, for all $y$ and $x \in H$.

Suppose moreover, that $\left[H, d \Pi_{\Lambda}\right]$ is an infinitesimally pseudo-unitary $U(\mathrm{G}(n))$-module with respect to $A$. Then $T$ is an intertwining map from this $U(\mathbf{G}(n))$-module into $\left[d C_{\mu}(K(n), H), d \Pi_{\bar{\Lambda}^{-1} \mathrm{P} 2}\right]$.

REMARK. Note, that the character $\bar{\Lambda}^{-1} \mathrm{P}^{2}$ is equal to the Weyl-conjugate character of $\Lambda$ only if $\Lambda$ is real.

Proof of the lemma. Fix $x \in H$. Then the map $f_{x}: y \rightarrow A(x, y)$ is conjugate linear. It is obviously $K(n)$-finite, and hence in $\bar{H}_{F}$. Therefore, we 
may apply Lemma 8. Accordingly, the map $f_{x} \rightarrow x_{f_{x}}$ is linear, and writing $x \rightarrow T x$ for the composition of the linear maps $x \rightarrow A(x, \cdot)=f_{x}$ with $f_{x} \rightarrow$ $x_{f_{x}}$, we get a linear map $T: H \rightarrow H$. Thus from Lemma 8 we have

$$
A(x, y)=f_{x}(y)=\left\langle x_{f_{x}}, y\right\rangle=\langle T x, y\rangle,
$$

for all $x, y \in H$.

Next, we prove the second statement of the lemma. By Theorem 8 of [10], we have for all $x, y \in H$ and $g \in G(n)$,

$$
\left\langle\Pi_{\bar{\Lambda}^{-1} \mathrm{P}^{2}}(g) x, y\right\rangle=\left\langle x, \Pi_{\Lambda}\left(g^{-1}\right) y\right\rangle .
$$

Let $Y \in \mathbf{G}(n)$. Then by differentiating the above equation along the one parameter subgroup $t \rightarrow \exp t Y$, we obtain:

$$
\left\langle d \Pi_{\bar{\Lambda}^{-1} \mathbf{P}^{2}}(Y) x, y\right\rangle=-\left\langle x, d \Pi_{\Lambda}(Y) y\right\rangle,
$$

for all $x$ and $y \in H$.

Now assume that $\left[H, d \Pi_{\Lambda}\right]$ is infinitesimally pseudo-unitary with respect to $A$. Then we have for all $x, y \in H$ and $Y \in \mathbf{G}(n)$,

$$
\begin{aligned}
\left\langle T d \Pi_{\Lambda}(Y) x, y\right\rangle & =A\left(d \Pi_{\Lambda}(Y) x, y\right)=-A\left(x, d \Pi_{\Lambda}(Y) y\right) \\
& =-\left\langle T x, d \Pi_{\Lambda}(Y) y\right\rangle=\left\langle d \Pi_{\bar{\Lambda}^{-1} P^{2}}(Y) T x, y\right\rangle .
\end{aligned}
$$

By the nondegeneracy of the form $\langle$,$\rangle , we have for all x \in H, \quad T d \Pi_{\Lambda}(Y) x=$ $d \Pi_{\bar{\Lambda}-1 P^{2}}(Y) T x$. Q.E.D.

COROLlaRY 4. In order that $\left[H, d \Pi_{\Lambda}\right]$ be infinitesimally pseudo-unitary it is necessary that either $\bar{\Lambda}^{-1} \mathrm{P}^{2}=\Lambda$, or that $\bar{\Lambda}=\Lambda$ and $\mu=\bar{\mu}$. In the first case $\nu$ is imaginary, and in the second case $\nu$ is real.

Proof. The proof is immediate from the definitions, the above lemma, and Lemma 3.

It now becomes a routine matter to list all the pseudo-unitary representations by making use of Theorem 1 . We will not do this here, but turn now to the classification of the unitary representations.

7. The unitary representations. Let $A$ be an Hermitian form on $H$. Suppose that the $U(\mathbf{G}(n))$-module $\left[H, d \Pi_{\Lambda}\right]$ is infinitesimally pseudo-unitary with respect to $A$. A key point in our argument is the following simple observation.

LEMMA 10. Under the above assumptions, $A$ is positive definite, if and only if $A$ is positive definite on each nontrivial subspace $E_{\omega} H,[\omega] \in \Omega_{\mu}(K(n))$.

Proof. The "only if" part is obvious. The "if" part follows immediately 
from the following observation. If $x \in E_{\omega} H$, and $y \in E_{\omega} \cdot H$, and if $[\omega] \neq$ $\left[\omega^{\prime}\right]$, then $A(x, y)=0$. However, this observation follows from the fact that the $K(n)$-representation $k \rightarrow R(k)=\Pi_{\Lambda}(k)$ on $H$ is unitary with respect to $A$, and the following computation:

Let $x \in E_{\omega} H$, and $y \in E_{\omega^{\prime}} H$ with $[\omega] \neq\left[\omega^{\prime}\right]$. Then

$$
\begin{aligned}
A(x, y) & =A\left(E_{\omega} x, E_{\omega^{\prime}} y\right)=\int_{K(n)} \bar{\chi}_{\omega}(k) A\left(R(k) x, E_{\omega^{\prime}} y\right) \\
& =\int_{K(n)} \bar{\chi}_{\omega}(k) A(x, R(k) y)=A\left(x, E_{\omega} E_{\omega^{\prime}} y\right)=0 \text {. Q.E.D. }
\end{aligned}
$$

We call an infinitesimal intertwining map $T$ positive definite, if the corresponding Hermitian form: $(x, y) \rightarrow\langle T x, y\rangle$ is positive definite. It is clear from the above lemma that an infinitesimal intertwining map is positive definite, if and only if the coefficient in each of its Fourier components is positive.

The next lemma is basic and concerns the positivity of the factors which occur in the definition of the maps $T_{n}(\mu, \nu, \omega)$. In accordance with Corollary 4, we need only consider the case of $\nu$ real. Hence we now assume that $\nu$ is real.

LEMMA 11. Let $i$ be an integer index with $1 \leqslant i \leqslant p$.

(1) First, assume that $n \geqslant 3$, and that if $n=2 p$, then $i \neq p$. Then the factor $\tau_{n i}\left(\mu, \nu, m_{i}\right)$ is positive for all positive integers $m_{i}$ which satisfy conditions $3 \mathrm{a}, \mathrm{b}$, if and only if

$$
0 \leqslant|\nu|<\left|\Lambda_{\mu i}\right|+(n+1) / 2-i .
$$

(Recall that the integer $m_{i}$ satisfies conditions $3 \mathrm{a}, \mathrm{b}$ if and only if $m_{i}+\Lambda_{\mu i}$ is the ith component of a $\mu$-admissible highest weight of $K(n)$.) Suppose $n_{i}$ and $n_{i}+1$ satisfy condition $3 \mathrm{a}, \mathrm{b}$, and suppose that $\nu=-\left(\Lambda_{\mu i}+(n+1) / 2-i+n_{i}\right)$. Then $\tau_{n i}\left(\mu, \nu, m_{i}\right) \geqslant 0$, for all $m_{i}$ which satisfy $3 \mathrm{a}, \mathrm{b}$.

(2) Let $n=2 p$, and $i=p$. Assume condition $\mathrm{C}$ of $\S 3$ is satisfied. Then the factor $\tau_{n p}\left(\mu, \nu, m_{p}\right)$ is never zero for any integer $m_{p}$ satisfying condition 3b. Moreover, this factor is positive for all $m_{p}$ satisfying $3 \mathrm{~b}$, if and only if $2 \Lambda_{\mu p-1}$ is an even integer, and $0 \leqslant|\nu|<1 / 2$, when $n \geqslant 4$, and if and only if $\mu=+$ and $0<|\nu|<1 / 2$, when $n=2$.

(3) Let $n=2 p$ and $i=p$. Assume condition $\mathrm{C}$ is violated. Then as usual, we consider two cases:

Case 1. $\nu=\left(n_{p}-n_{p}^{\prime}\right) / 2 \leqslant 0$. (We use the notation of $\S 3$.) Then the factors $\tau_{n p}^{ \pm}\left(\mu, \nu, m_{p}\right)$ are defined, and are positive or zero for all $m_{p}$ satisfying $3 \mathrm{~b}$. (The zeros are given by Lemma 6, or Lemma 7 in case $p=1$.)

Case 2. $\nu=\left(n_{p}-n_{p}^{\prime}\right) / 2>0$. Then the factor $\tau_{n p}^{F}\left(\mu, \nu, m_{p}\right)$ is defined for all $m_{p}$ satisfying $3 \mathrm{~b}$. It is positive or zero for all such $m_{p}$, if and only if 
$n_{p}=n_{p}^{\prime}+1$; hence $\nu=1 / 2$ and $\Lambda_{\mu p-1}$ is an integer. In case $n=2$, the last condition must be replaced by the condition that $\mu=+$, and $\nu=1 / 2$.

Proof of Statement 1. If $i>1$, and if $\Lambda_{\mu i-1}=\Lambda_{\mu i}$, then every $\mu$-admissible class $[\omega]$ must have the property that $\Lambda_{\mu i}=\Lambda_{\omega i}$. Consequently, in this case $m_{i}=0$ is the only integer for which $\tau_{n i}\left(\mu, \nu, m_{i}\right)$ is defined. This factor is by definition equal to 1 . Now, if $i=1$, or if $\Lambda_{\mu i} \neq \Lambda_{\mu i-1}$, then there is more than one integer $m_{i}$ which satisfies the conditions $3 \mathrm{a}, \mathrm{b}$. It follows from equation (17) that for all these integers, $\tau_{n i}\left(\mu, \nu, m_{i}\right)>0$, if and only if

$$
\frac{\left|\Lambda_{\mu i}\right|+(n+1) / 2-i+m_{i}-\nu}{\left|\Lambda_{\mu i}\right|+(n+1) / 2-i+m_{i}+\nu}>0,
$$

for all integers $m_{i}$ which satisfy the conditions $3 \mathrm{a}, \mathrm{b}$. (We remark again, that the absolute value sign is needed only in the case where $n=2 p+1$ and $i=p$.) The condition (*) is equivalent to the condition that

$$
\left(\left|\Lambda_{\mu i}\right|+(n+1) / 2-i+m_{i}\right)^{2}>\nu^{2} .
$$

The left-hand side of the last inequality attains its minimum for $m_{i}=0$. Similarly, the condition that $\tau_{n i}\left(\mu, v, m_{i}\right) \geqslant 0$ for all $m_{i}>\dot{n}_{i}$, and satisfying $3 \mathrm{a}, \mathrm{b}$ is equivalent to the condition that

$$
\left(\left|\Lambda_{\mu i}\right|+(n+1) / 2-i+m_{i}\right)^{2} \geqslant \nu^{2},
$$

which under the second hypothesis of statement 1 is satisfied automatically for $m_{i} \geqslant n_{i}+1$. For $m_{i} \leqslant n_{i}, \tau_{n i}\left(\mu, \nu, m_{i}\right)=0$, by Lemma 4 . The statement follows.

Proof of Statement 2. Let $n=2 p$ and $i=p$. First suppose that $n \geqslant 4$. If $\Lambda_{\mu p-1}=0$, then $m_{p}=0$ is the only integer for which the factor $\tau_{n p}\left(\mu, \nu, m_{p}\right)$ is defined, and by definition, this factor is equal to 1 . Now suppose that $\Lambda_{\mu p-1}>0$. Then it follows from equation (5) that $\tau_{n p}\left(\mu, \nu, m_{p}\right)>0$, for all $m_{p}$ for which this factor is defined (inequality $3 b$ ) if

$$
\left(-\Lambda_{\mu p-1}+1 / 2+m_{p}\right)^{2}>v^{2}
$$

for all integers $m_{p}$ which satisfy the inequalities $3 \mathrm{~b}$. Now if $2 \Lambda_{\mu p-1}$ is an odd integer, then one possibility for $m_{p}$ is $m_{p}=\Lambda_{\mu p-1}-1 / 2$. For this integer we have $0>v^{2}$, which is impossible. Hence, $2 \Lambda_{\mu p-1}$ must be an even integer. In this case the minimum of the left-hand side of inequality $(* * *)$ is attained when $m_{p}=\Lambda_{\mu p-1}$. Hence statement 2 follows for this case.

Next assume that $n=2$. If $\mu=-$, we find from equation 10 that $\tau_{21}(-, \nu,-1)=(-\nu) / \nu=-1$, for all $\nu \neq 0$. Hence, we must have $\mu=+$. On 
the other hand, we see that $\tau_{21}(+, \mu, m)>0$ for all $m \in \mathbf{Z}$, if and only if $(j+1 / 2)^{2}-\nu^{2}>0$ for all $j \in \mathbf{Z}$. Hence, the statement follows in this case as well.

Statement 3 follows easily from the explicit formulas given in equations (7) and (8), in case $n \geqslant 4$, and from equations (12), (13), (14), and (15), in the case when $n=2$.

From our point of view the irreducible unitary representations fall into two broad classes I and II:

I. The principal series. These representations are the representations on $L_{\mu}^{2}(K(n), H)$ with $[H, \mu]$ an arbitrary irreducible finite-dimensional representation of $M(n)$, and $\nu$ pure imaginary or zero. Note that it follows from the irreducibility conditions of $[10$, Theorem 6$]$, or in $\S 5$, that the only nonirreducible case of the principal series occurs if $\nu=0, n=2 p \geqslant 4$, and the component $\Lambda_{\mu p-1}$ is a half odd integer, or in case $n=2, \nu=0$, and $\mu$ is the nontrivial representation of $M(2)$. In these cases the principal series splits into two irreducible components.

II. Representations with a redefined inner product on $d C_{\mu}(K(n), H)$. In this case one defines an inner product on $d C_{\mu}(K(n), H)$ such that for some character $\Lambda$ on $S(n)$ the representation $\left[d C_{\mu}(K(n), H), d \Pi_{\Lambda}\right]$ is infinitesimally unitary with respect to this inner product. Then the unitary representation of $G(n)$ is supposed to act on the Hilbert space completion, with respect to the norm defined by this inner product, of the quotient space $d C_{\mu}(K(n), \mathrm{H}) /($ radical $(A))$, where $A$ is the inner product. This second class of representations splits up into two subclasses:

IIA. Irreducible complementary series. In this case the radical of the inner product $A$ is equal to the zero space.

IIB. Nonirreducible case; the end point and isolated point representations. In this case the radical of $A$ is nontrivial.

First, we discuss the class IIA in more detail. For this class there is no substantial difference between the case $n=2 p$ and the case $n=2 p+1$. By Corollary 4, we must have $\mu=\bar{\mu}$, and $\nu$ a real number, where $\nu=d \Lambda(H)-$ $(n-1) / 2$. By Lemma 9, the inner product $A$ which makes $\left[d C_{\mu}(K(n), H), d \Pi_{\Lambda}\right]$ infinitesimally unitary can be given as follows:

$$
(x, y) \rightarrow A_{n}(\mu, \nu ; x, y)=\left\langle T_{n}(\mu, \nu) x, y\right\rangle .
$$

By Lemma 10, this Hermitian form is positive definite provided that the scalar $t_{n}(\mu, \nu, \omega)$ is positive for each $\mu$-admissible class [ $\left.\omega\right]$. By formula (20) in $\S 3$, this last condition is equivalent to the condition that each of the factors $\tau_{n i}\left(\mu, \nu, m_{i}\right)$ is positive for $1 \leqslant i \leqslant p$, and $m_{i}$ given by $m_{i}=\Lambda_{\omega i}-\Lambda_{\mu i}$, if 
$1 \leqslant i<p$, and $m_{p}=\Lambda_{\omega p}-\left|\Lambda_{\mu p}\right|$, if $n=2 p+1$, or $m_{p}=\Lambda_{\omega p}-\Lambda_{\omega p-1}$, if $n=2 p$.

Now assume first that $n=2 p+1$. Then the condition that $\mu=\bar{\mu}$ implies that the component $\Lambda_{\mu p}=0$. Suppose that there is an index $j$ such that $1 \leqslant j<p$, and such that $\Lambda_{\mu p}=0$. Then $0=\Lambda_{\mu j}=\Lambda_{\mu j+1}=\cdots=\Lambda_{\mu p}$. Then only the integers $m_{j+1}=\cdots=m_{p}=0$ are possible. In this case, the factors $\tau_{j+1}(\mu, \nu, 0)=\cdots=\tau_{p}(\mu, \nu, 0)=1$. Let $j$ be the first integer such that $\Lambda_{\mu j}=0$. Then by statement 1 of Lemma 11 we find that the positivity condition becomes

$$
|\nu| \in \bigcap_{i=1}^{j}\left[0, \Lambda_{\mu i}+(n+1) / 2-i\right)=[0,(n+1) / 2-j) .
$$

This condition is both necessary and sufficient for the component $t_{n}(\mu, \nu, \omega)$. to be positive for each $\mu$-admissible class $[\omega] \in \Omega_{\mu}(K(n))$.

Now let $n=2 p$. If $\Lambda_{\mu p-1}=0$, then as before, we let $j$ be the smallest integer such that $\Lambda_{\mu j}=0$, and we have as before, the positivity condition:

$$
|v| \in \bigcap_{i=1}^{j}\left[0, \Lambda_{\mu i}+(n+1) / 2-i\right)=[0,(n+1) / 2-j) .
$$

That is, the above condition is both necessary and sufficient for the Hermitian form $A_{n}(\mu, \nu ; \cdot, \cdot)$ to be positive definite. Now assume that $\Lambda_{\mu p-1} \neq 0$. Then by statement 2 of Lemma 11 and the above reasoning we must have that $2 \Lambda_{\mu p-1}$ is an even integer and that $|\nu| \in[0,1 / 2)$. Now this interval is contained in the interval

$$
\bigcap_{i=1}^{p-1}\left[0, \Lambda_{\mu i}+(n+1) / 2-i\right)
$$

Hence, the condition that $|\nu| \in[0,1 / 2)$ and $\Lambda_{\mu p-1} \in Z$ is both necessary and sufficient for the positiveness of $A_{n}(\mu, \nu ; \cdot, \cdot)$, when $\Lambda_{\mu p-1} \neq 0$.

REMARK 1. If $n=2 p$, then the above analysis implies that one cannot have a complementary series if $[H, \mu]$ is a faithful representation of $M(n)$.

Now assume that the above conditions for positive definiteness are satisfied. Thus we assume that $A_{n}(\mu, \nu ; \cdot, \cdot)$ is a positive definite inner product on the space $d C_{\mu}(K(n), H)$. Let $H_{\mu \nu}$ denote the Hilbert space completion of this space with respect to the Hilbert space norm defined by this inner product. We wish to argue that there exists a globally defined unitary representation of $G(n)$ whose differential is the infinitesimally unitary representation $\left[d C_{\mu}(K(n), H), d \Pi_{\Lambda}\right]$. For this purpose, we may invoke a theorem of Nelson (see the discussion below). However, we shall give a simpler argument which is valid in the case of the complementary series, and which generalizes an argument due to Bargmann [1, p. 619]. 
Proposition 1. Let $\nu \geqslant 0$, and assume that $\nu$ satisfies one of the above conditions for the positive definiteness of the form $A_{n}(\mu, \nu ; \cdot, \cdot)$. The $T_{n}(\mu, \nu)$ may be extended to a bounded positive operator on $L_{\mu}^{2}(K(n), H)$ with operator norm $\left\|T_{n}(\mu, \nu)\right\| \leqslant 1$. The last inequality is strict unless $\nu=0$. Hence, $L_{\mu}^{2}(K(n), H)$ may be embedded as a dense subspace of $H_{\mu \nu}$.

Proof. An examination of the formulas (17), or the formulas (4) and (5) shows that $t_{n}(\mu, \nu, \omega) \leqslant 1$ for all $[\omega] \in \Omega_{\mu}(K(n))$. The inequality is strict unless $\nu=0$. Now $\left\{E_{\omega}:[\omega] \in \Omega_{\mu}(K(n))\right\}$ is a complete orthogonal family of projections on the Hilbert space $L_{\mu}^{2}(K(n), H)$. Hence, the proposition follows directly from the definition of the operator $T_{n}(\mu, \nu)$. Q.E.D.

Now since the action of $G(n)$ is already globally defined on the space $L_{\mu}^{2}(K(n), H)$, by $\Pi_{\Lambda}$, this action extends, by continuity, to an action of $G(n)$ on the Hilbert space completion $H_{\mu \nu}$.

If the conditions for positive definiteness of the form $A_{n}(\mu, \nu)$ are satisfied, but now $\nu \leqslant 0$, then we note that $\left[L_{\mu}^{2}(K(n), H), \Pi_{\Lambda^{\prime}}\right]$ is infinitesimally equivalent to the representation $\left[L_{\mu}^{2}(K(n), H), \Pi_{\Lambda}\right]$. Hence, by the above argument, this case is unitarizable as well.

Next, we turn to a discussion of the case IIB. Again, by Lemma 9, we may assume that the inner product $A$ is given by:

$$
(x, y) \rightarrow A_{n}(\mu, \nu ; x, y)=\left\langle T_{n}(\mu, \nu) x, y\right\rangle,
$$

where now the radical of $A_{n}(\mu, \nu ; \cdot, \cdot)$, which is the same as the kernel of $T_{n}(\mu, \nu)$, is assumed to be nontrivial. (In case $n=2 p$, and in case the operators $T_{n}^{ \pm}(\mu, \nu)$ of $T_{n}^{F}(\mu, \nu)$ are defined, we use the notation $A_{n}^{ \pm}(\mu, \nu ; \cdot, \cdot)$ or $A_{n}^{F}(\mu, \nu ; \cdot, \cdot)$ to denote the corresponding inner products.) By the discussion of Theorem 2 in $\S 5$, and by the fact that $\mu=\bar{\mu}$, we have that $T_{n}(\mu, \nu)$ is one to one on its range. Hence, if $T_{n}(\mu, \nu)$ is positive semidefinite, then $A_{n}(\mu, \nu ; \cdot, \cdot)$ is positive definite on range $\left(T_{n}(\mu, \nu)\right)$. Now, by Theorem 2 , condition D of $\S 5$ must be violated. Accordingly, we first assume that

$$
\nu=\left(\Lambda_{\mu i}+(n+1) / 2-i+n_{i}\right),
$$

for some index $i$ such that $1 \leqslant i \leqslant p$, and we first assume that if $n=2 p$, then $i \neq p$. (The case with $n=2 p$ and $i=p$ will be handled separately.) Here $n_{i}$ is a nonnegative integer such that $\Lambda_{\omega i}=n_{i}+\Lambda_{\mu i}$ for some $\mu$-admissible class $[\omega]$, and $1+n_{i}+\Lambda_{\mu i}$ is also assumed to be the $i$ th component of some $\mu$-admissible class. By Lemma 11 , statement 1 , if $j>i$, then the factors $\tau_{n j}\left(\mu, \pm \nu, m_{i}\right)$ cannot all be positive, unless there is only one, namely $\tau_{n j}(\mu, \pm \nu, 0)$. Thus, $0=\Lambda_{\mu i}=\Lambda_{\mu i+1}=\cdots$. On the other hand, if $i \neq 1$, then we must have $\Lambda_{\mu j} \neq 0$, for $j<i$. (Otherwise, we would have $\Lambda_{\omega i}=0$ for all $\mu$-admissible 
classes $[\omega]$.$) For such indices j$, the condition of statement 1 in Lemma 11 is satisfied automatically. Hence, the positivity of $t_{n}(\mu, \pm \nu, \omega)$ hinges upon the positivity of the factor $\tau_{n i}\left(\mu, \pm \nu, m_{i}\right)$ for each integer $m_{i}$ for which this factor is defined.

Now by Lemma 11, statement 2 , and Lemma $4 \mathrm{c}$, we have $\tau_{n i}\left(\mu,-\nu, m_{i}\right)=$ 0 , for $0 \leqslant m_{i} \leqslant n_{i}$, while $\tau_{n i}\left(\mu,-\nu, m_{i}\right)>0$, for $n_{i}<m_{i}$. On the other hand, assume that $n_{i}>0$. Then we have $\tau_{n i}(\mu, \nu, 0)=1$. However, $\tau_{n i}(\mu, \nu, 1)=$ $-n_{i} /\left(n+1-2 i+n_{i}\right)<0$, as can be established by substitution into the formula (4) or the formula (17). Hence, $T_{n}(\mu, \nu)$ is not positive unless $n_{i}=0$. By Theorem $2, \operatorname{ker}\left(T_{n}(\mu,-\nu)\right)=\operatorname{range}\left(D_{\lambda^{\prime}}^{-}\right)$, while $\operatorname{ker}\left(T_{n}(\mu, \nu)\right)=\operatorname{range}\left(D_{\lambda}^{+}\right)$. Thus we have established the following result.

If $\nu=(n+1) / 2-i+n_{i}$, and the above stated conditions on the index $i$ and the integer $n_{i}$ hold, then the quotient $U(\mathrm{G}(n))$-module

$$
\left[d C_{\mu}(K(n), H), d \Pi_{\Lambda^{\prime}}\right] /\left[D_{\Lambda^{\prime}}^{-} d C_{\mu}(K(n), H), d \Pi_{\Lambda^{\prime}}\right]
$$

is infinitesimally unitary with respect to the inner product $(x, y) \rightarrow A_{n}(\mu,-v ; x, y)$ $=\left\langle T_{n}(\mu,-v) x, y\right\rangle$. While the "supplementary" quotient module

$$
\left[d C_{\mu}(K(n), H), d \Pi_{\Lambda}\right] /\left[D_{\lambda}^{+} d C_{\mu}(K(n), H), d \Pi_{\Lambda}\right]
$$

is infinitesimally unitary with respect to the inner product $(x, y) \rightarrow A_{n}(\mu, \nu ; x, y)$ $=\left\langle T_{n}(\mu, \nu) x, y\right\rangle$, if and only if $n_{i}=0$.

REMARK 2. The quotient module $\left[d C_{\mu}(K(n), H), d \Pi_{\Lambda^{\prime}}\right] /\left[D_{\lambda^{\prime}}^{-} d C_{\mu}(K(n), H), d \Pi_{\Lambda^{\prime}}\right]$ is infinitesimally equivalent to the submodule $\left[D_{\lambda}^{+} d C_{\mu}(K(n), H), d \Pi_{\Lambda}\right]$ (see $[10$, Theorem 6]). This infinitesimal equivalence can be implemented directly with the map $T_{n}(\mu,-\nu)$ (see Theorem 2). This submodule can be made infinitesimally unitary directly with the inner product defined by $(x, y) \rightarrow B_{n}(\mu,-\nu ; x, y)=$ $\left\langle T_{n}(\mu,-\nu)^{-1} x, y\right\rangle$, where $x, y \in$ range $\left(D^{+}\right)$, and $T_{n}(\mu,-\nu)^{-1}$ is the inverse of $T_{n}(\mu,-\nu)$ defined only on this range. It is clear that $B_{n}(\mu,-\nu ; \cdot, \cdot)$ is positive definite. Moreover, it follows from the intertwining property of $T_{n}(\mu,-v)$ that for all $Y \in \mathrm{G}(n)$, and $x \in \operatorname{range}\left(D_{\lambda}^{+}\right)$,

$$
T_{n}(\mu,-\nu)^{-1} d \Pi_{\Lambda}(Y) x=d \Pi_{\Lambda^{\prime}}(Y) T_{n}(\mu,-\nu)^{-1} x, \quad \text { modulo }\left(1-D_{\lambda}^{+}=D_{\lambda^{\prime}}^{-}\right) .
$$

The skew-symmetry of the representation follows as in the calculation in the proof of Lemma 9.

Similarly, if $n_{i}=0$, the $U(\mathbf{G}(n))$-module [range $\left(D_{\lambda^{\prime}}^{-}\right), d \Pi_{\Lambda^{\prime}}$ ] can be made infinitesimally unitary directly by defining on the range of $D_{\lambda^{\prime}}^{-}$an inner product $B_{n}(\mu, \nu ; \cdot, \cdot)$ by the formula

$$
B_{n}(\mu, \nu ; x, y)=\left\langle T_{n}(\mu, \nu)^{-1} x, y\right\rangle,
$$


for all $x, y \in$ range $\left(D_{\lambda^{\prime}}^{-}\right)$. Again, the map $T_{n}(\mu, \nu)^{-1}$ is the inverse of $T_{n}(\mu, \nu)$ defined only on the range of $T_{n}(\mu, \nu)$, namely $D_{\lambda^{\prime}}^{-}$.

REMARK 3. In the nonsingular cases, namely when $\operatorname{ker}\left(T_{n}(\mu, \nu)\right)=\{0\}$, we have the following simple formula for the inverse of $T_{n}(\mu, \nu): T_{n}(\mu, \nu)^{-1}=$ $T_{n}(\mu,-\nu)$. This formula can be established easily from the definition in formulas (19) and (20), and from the definitions of the factors $\tau_{n j}\left(\mu, \nu, m_{j}\right)$.

REMARK 4. In case $n_{i}=0$ we have explicitly:

$$
D_{\lambda^{\prime}}^{-}=\left\{E_{\omega}:[\omega] \in \Omega_{\mu}(K(n)), \Lambda_{\omega i}=0\right\} .
$$

Now, if $i=1$, then $\Lambda_{\mu}=0$. Hence, $[H, \mu]$ is the trivial representation of the subgroup $M(n)$ on $\mathbf{C}$. In this special case, $D_{\lambda^{\prime}}^{-}$reduces to the projection onto the subspace of constant functions, and the corresponding unitary representation is the trivial unitary representation of $G(n)$.

Now we turn to the case when $n=2 p$, and $i=p$. The discussion is similar to the above one, except now we must use statement 3 of Lemma 11 to determine the positivity conditions for the factors $\tau_{n p}\left(\mu, \nu, m_{p}\right)$ which occur in the definition of the intertwining maps.

First, we assume that Case $1^{\prime}$ of Theorem 2 holds. This case is equivalent to the Case 1 in Lemma 11 for the parameter $-\nu$. Thus, we have $\nu=-s-1 / 2 \geqslant 0$, and $-s>s$. If $n \geqslant 4$, then all the factors $\tau_{n j}\left(\mu, \nu, m_{j}\right)$ for $j<p$ are positive because of statement 1 of Lemma 11, and because of the estimate:

$$
\begin{aligned}
0 & \leqslant|\nu|=|s+1 / 2| \leqslant \Lambda_{\mu p-1}+1 / 2 \\
& <\Lambda_{\mu p-1}+(n-1) / 2-j \leqslant \Lambda_{\mu j}+(n+1) / 2-j .
\end{aligned}
$$

It follows from Lemma 11 and from Theorem 2 that $T_{n}^{ \pm}(\mu,-\nu)$ are positive operators with kernels given by range $\left(D_{\lambda^{\prime} p}^{\mp}\right)$. Hence, these operators are positive definite on the ranges of the projections $D_{\lambda p}^{ \pm}$. Hence, in particular, the inverses $T_{n}^{ \pm}(\mu,-\nu)^{-1}$ are defined on the ranges of the projections $D_{\lambda p}^{ \pm}$. Now, from Theorem 2 , the quotient modules $\left[d C_{\mu}(K(n), H), d \Pi_{\Lambda^{\prime}}\right] /\left[\operatorname{range}\left(D_{\lambda^{\prime} p}^{\mp}\right), d \Pi_{\Lambda}\right]$ are isomorphic, via the maps $T_{n}^{ \pm}(\mu,-v)$, to the submodules [range $\left(D_{\lambda p}^{ \pm}\right), d \Pi_{\Lambda}$ ]. (Throughout this discussion the upper signs go with upper signs, and the lower signs go with the lower signs.) By an argument similar to the above one, the above quotient modules are infinitesimally unitary with respect to the inner products $(x, y) \rightarrow A_{n}(\mu,-\nu)=\left\langle T_{n}(\mu,-\nu) x, y\right\rangle$. Then it follows from an argument similar to the one in Remark 2, that the above subrepresentations are infinitesimally unitary with respect to the inner product $(x, y) \rightarrow B_{n}(\mu,-v ; x, y)=\left\langle T_{n}(\mu,-\nu)^{-1} x, y\right\rangle$.

Now let us assume that Case $2^{\prime}$ of Theorem 2 holds. Equivalently, Case 2 with $\nu$ replaced by $-\nu$ holds. Then, by the above discussion and by statement 3 of Lemma $11, T^{F}(\mu,-\nu)$ is a positive operator if and only if there is precisely 
one factor $\tau_{n p}^{F}\left(\mu,-\nu, m_{p}\right)$ different from zero. This situation can only occur if $\nu=-1 / 2$, and $n_{p}=n_{p}^{\prime}+1$ (see $\S 3$ ). We must then have $\Lambda_{\mu p-1}=n_{p}^{\prime}$, in case $n \geqslant 4$. Thus $\Lambda_{\mu p-1}$ must be a nonnegative integer. If $n=2$, this last condition must be replaced by the condition that $\mu$ is the trivial representation of $M(2)$.

Now assume that these conditions hold. Then $T_{n}^{F}(\mu,-\nu)$ is positive, and is positive definite on range $\left(D_{\lambda p}^{F}\right)$. Moreover, by Theorem 2, we have $\operatorname{ker}\left(T_{n}^{F}(\mu,-\nu)\right)=$ range $\left(D_{\lambda^{\prime} p}^{+}+D_{\lambda^{\prime} p}^{-}\right)$. Then by arguments similar to the above ones, we have that $\left[d C_{\mu}(K(n), H), d \Pi_{\Lambda^{\prime}}\right] /\left(\operatorname{range}\left(D_{\lambda^{\prime} p}^{+}+D_{\lambda^{\prime} p}^{-}\right), d \Pi_{\Lambda^{\prime}}\right]$ is infinitesimally unitary with respect to the inner product $(x, y) \rightarrow A_{n}^{F}(\mu,-\nu ; x, y)=$ $\left\langle T_{n}(\mu,-v) x, y\right\rangle$. Then the subrepresentation [range $\left.\left(D_{\lambda p}^{F}\right), d \Pi_{\Lambda}\right]$, which is equivalent to the above quotient module, is infinitesimally unitary with respect to the inner product $(x, y) \rightarrow B_{n}(\mu,-\nu ; x, y)=\left\langle T_{n}(\mu,-\nu)^{-1} x, y\right\rangle$.

In order to prove that there exist globally defined unitary group representations for each of the infinitesimally unitary representations listed above for Case IIB, one could argue as with Proposition 1 above. A modification of this argument is valid for the above representations in the subrepresentation picture rather than in the quotient picture. However we shall instead apply a theorem of Nelson [6, Theorem 5]. According to the hypotheses of that theorem it will be sufficient to show that for each of the representations listed above, the differential operator $d \Pi_{\Lambda}(\nabla)$, with

$$
\nabla=\sum_{i=2}^{n} Y_{0 i}^{2}+H^{2}+\sum_{i=1}^{n-1} \sum_{j=2}^{n} X_{i j}^{2}=\Omega+\Omega_{K},
$$

is essentially selfadjoint. Here $\Omega$ and $\Omega_{K}$ are the Casimir operators of $\mathbf{G}(n)$ and $\mathbf{K}(n)$, respectively, in the normalization of [10, Lemma 6]. However, this fact follows immediately from the fact that this operator is symmetric for any infinitesimally unitary representation, and from the fact that the ranges of the projections $E_{\omega},[\omega] \in \Omega_{\mu}(K(n))$, are eigenspaces of this operator.

By Theorem 8 of Harish-Chandra [3a], every infinitesimal equivalence class of quasi-simple representations contains at most one unitary equivalence class of unitary representations. Second, every irreducible unitary representation is quasisimple. Third, every irreducible quasi-simple representation is infinitesimally equivalent to a subrepresentation of a principal series representation (possibly nonunitary), by Corollary 2 . If we combine the above analysis with the computation of the eigenvalues of the Casimir operator in [10, Lemma 6], we have proved the following result.

THEOREM 3. Every irreducible unitary representation of $G(n)$ is unitarily equivalent to one of the following unitary representations. 
I. The irreducible principal series. Let $[H, \mu]$ be an irreducible finitedimensional representation of the subgroup $M(n)$. Let $\nu=d \Lambda(H)-(n-1) / 2$. The representations in this class are the representations $\left[L_{\Lambda}^{2}(K(n), H), \Pi_{\Lambda}\right]$ with $\nu$ pure imaginary, with $\nu \neq 0$, in case $n=2 p$, and $\mu$ is a faithful representation of $M(n)$. (The last condition is equivalent to the condition that $2 \Lambda_{\mu p-1}$ is odd, if $n \geqslant 4$, and $\mu$ is nontrivial if $n=2$.) We also have

$$
\begin{aligned}
d \Pi_{\Lambda}(\Omega) & =\nu^{2}-\frac{(n-1)^{2}}{4}+\sum_{i=1}^{p-1}\left(\Lambda_{\mu i}+n-2 i-1\right) \Lambda_{\mu i}, & & \text { if } n=2 p \geqslant 4, \\
& =\nu^{2}-1 / 4, & & \text { if } n=2, \\
& =\nu^{2}-\frac{(n-1)^{2}}{4}+\sum_{i=1}^{p}\left(\Lambda_{\mu i}+n-2 i-1\right) \Lambda_{\mu i}, & & \text { if } n=2 p+1 .
\end{aligned}
$$

IIA. The irreducible complementary series. Again, set $\nu=d \Lambda(H)-(n-1) / 2$. Let $[H, \mu]$ be an irreducible finite-dimensional representation of the subgroup $M(n)$. If $n=2 p+1$ then we must have $\Lambda_{\mu p}=0$. Let $j$ be the smallest integer such that $\Lambda_{\mu j}=0$. Then $\nu$ is real and $\nu \in(-(n+1) / 2+j,(n+1) / 2-$ j). Similarly, if $n=2 p \geqslant 4$, and if $\Lambda_{\mu p-1}=0$, let $j$ be the smallest integer such that $\Lambda_{\mu j}=0$. Then $\nu$ must be real and $\nu \in(-(n+1) / 2+j,(n+1) / 2-j)$. If $\Lambda_{\mu p-1} \neq 0$, then this component must be an integer, and $\nu$ is real and in the interval $(-1 / 2,1 / 2)$. If $n=2$, then $\mu$ must be the trivial representation of $M(2)$ and $\nu$ is real and in the interval $(-1 / 2,1 / 2)$. With the above conditions the form $(x, y) \rightarrow A_{n}(\mu, \nu ; x, y)=\left\langle T_{n}(\mu, \nu) x, y\right\rangle$ is positive definite on $d C_{\mu}(K(n), H)$. Let $H_{\mu \nu}$ denote the Hilbert space completion of $d C_{\mu}(K(n), H)$, with respect to the above form. There exists a unique unitary representation $U$ on this completion, and $d U=d \Pi_{\Lambda}$. Moreover,

$$
\begin{aligned}
d \Pi_{\Lambda}(\Omega) & =\nu^{2}-(n-1)^{2} / 4+\sum_{i=1}^{p-1}\left(\Lambda_{\mu i}+n-2 i-1\right) \Lambda_{\mu i}, & & \text { if } n>3, \\
& =v^{2}-1, & & \text { if } n=3, \\
& =v^{2}-1 / 4, & & \text { if } n=2 .
\end{aligned}
$$

The representations corresponding to the pairs $(\mu, \nu)$ and $(\mu,-\nu)$ are unitarily equivalent. Moreover, the complementary series as defined here overlaps with the principal series in case $\nu=0$. Here, the operator $T_{n}(\mu, 0)$ reduces to the identity, and $H_{\mu 0}=L_{\mu}^{2}(K(n), H)$.

IIB. Nonirreducible cases; end point and isolated point representations.

(1) Suppose $n=2 p+1$, and the irreducible representation $[H, \mu]$ has highest weight components $0=\Lambda_{\mu j}=\Lambda_{\mu j+1}=\cdots=\Lambda_{\mu}$, or suppose $n=2 p \geqslant 4$, and the representation $[H, \mu]$ has highest weight components $0=\Lambda_{\mu j}=\cdots=$ 
$\Lambda_{\mu p-1}$. Assume that $j$ is the smallest integer such that $\Lambda_{\mu j}=0$. Let $n_{j}$ be a nonnegative integer such that $\Lambda_{\mu j-1} \geqslant n_{j}$, in case $j \neq 1$. Then the inner product $(x, y) \rightarrow B_{n}(\mu,-\nu ; x, y)=\left\langle T_{n}(\mu,-\nu)^{-1} x, y\right\rangle$ is positive definite, with $\nu=$ $d \Lambda(H)-(n-1) / 2=(n+1) / 2-j+n_{j}$, on the space:

$$
D_{\lambda}^{+} d C_{\mu}(K(n), H)=\operatorname{span}\left\{E_{\omega} L_{\mu}(K(n), H): \Lambda_{\omega j} \geqslant n_{j}\right\} .
$$

Let $H_{\mu \nu}^{+}$denote the Hilbert space completion of the above space with respect to the norm defined by this inner product. Then there exists a uniquely defined unitary action of $G(n)$ on this space, and $d U=d \Pi_{\Lambda}$. The eigenvalue of $\Omega$ is

$$
d \Pi_{\Lambda}(\Omega)=\left(n_{j}+1-j\right)\left(n_{j}+n-j\right)+\sum_{i=1}^{j}\left(\Lambda_{\mu i}+n-2 i-1\right) \Lambda_{\mu i} .
$$

(2) Make the assumptions in 1 above. In addition, assume that $n_{j}=0$. Let $H_{\mu \nu}^{-}$be the Hilbert space completion of the space

$$
D_{\lambda^{\prime}}^{-} d C_{\mu}(K(n), H)=\operatorname{span}\left\{E_{\omega} L_{\mu}^{2}(K(n), H): \Lambda_{\omega j}=0\right\},
$$

with respect to the norm defined by the inner product $B_{n}(\mu, \nu ; \cdot, \cdot)$. The latter is positive definite, and is given by $(x, y) \rightarrow B_{n}(\mu, \nu ; x, y)=\left\langle T_{n}(\mu, \nu)^{-1} x, y\right\rangle$. The unitary action is uniquely defined, and for this action $U$ we have $d U=$ $d \Pi_{\Lambda^{\prime}}$, where $\Lambda^{\prime}$ is determined by $d \Lambda^{\prime}(H)=-\nu+(n-1) / 2=j-1$. The eigenvalue of $\Omega$ is obtained by substituting $n_{j}=0$ into the last formula for this eigenvalue. We remark that in case $j=1$, then $\mu$ is the trivial representation of $M(n)$, and $U$ is a one-dimensional representation on the space of constant functions.

(3) Now assume that $n=2 p$ and $\nu=-1 / 2$. If $n \geqslant 4$ assume that $\Lambda_{\mu p-1}$ is a nonnegative integer. If $n=2$ assume that $\mu$ is the trivial representation of $M(2)$. (Thus, in both cases, $[H, \mu]$ is not a faithful representation of the subgroup $M(n)$.) Let $H_{\mu^{1 / 2}}^{F}$ be the Hilbert space completion of the space

$$
D_{p-1, p}^{F} d C_{\mu}(K(n), H)=\operatorname{span}\left\{E_{\omega} L_{\mu}^{2}(K(n), H): \Lambda_{\omega p}=0\right\}
$$

with respect to the norm defined by the inner product $B_{n}(\mu, 1 / 2 ; \cdot, \cdot)$. The latter is positive definite, and is defined by $(x, y) \rightarrow B_{n}(\mu, \nu ; x, y)=\left\langle T_{n}^{F}(\mu, 1 / 2)^{-1} x, y\right\rangle$. Then there is a uniquely defined unitary action $U$ on this space with $d U=$ $d \Pi_{\Lambda}$, with $d \Lambda(H)=-1 / 2+(n-1) / 2=n / 2-1$, and

$$
\begin{aligned}
d \Pi_{\Lambda}(\Omega) & =1 / 2 n(1 / 2 n-1)+\sum_{i=1}^{p-1} \Lambda_{\mu i}\left(\Lambda_{\mu i}+n-2 i-1\right), & & \text { if } n=2 p \geqslant 4, \\
& =0, & & \text { if } n=2 .
\end{aligned}
$$


We remark that in case $n=2$, this representation is the one-dimensional representation of $G(2)$ on the space of constant functions.

(4) If $n=2 p$, let $[H, \mu]$ be an irreducible representation of $M(n)$ such that, in case $n \geqslant 4, \Lambda_{\mu p-1} \neq 0$. Write $\nu=-s-1 / 2$ Assume $\nu \geqslant 0$, and that $s$ is a number such that $-\Lambda_{\mu p-1} \leqslant s \leqslant \Lambda_{\mu p-1}$, and $s-\Lambda_{\mu p-1} \in Z$, if $n \geqslant$ 4 , and if $n=2,2 s$ is an even (odd) integer if $\mu$ is trivial (nontrivial). Then let $H_{\mu \nu}^{ \pm}$be the Hilbert space completions of the spaces

$$
\begin{aligned}
& D_{\lambda p}^{+} d C_{\mu}(K(n), H)=\operatorname{span}\left\{E_{\omega} L_{\mu}(K(n), H): \Lambda_{\omega p} \geqslant-s\right\}, \\
& D_{\lambda p}^{-} d C_{\mu}(K(n), H)=\operatorname{span}\left\{E_{\omega} L_{\mu}(K(n), H): \Lambda_{\omega p} \leqslant s\right\}
\end{aligned}
$$

with respect to the norm defined by the inner products $B_{n}^{ \pm}(\mu,-\nu ; \cdot, \cdot)$. The latter is positive definite on space $D_{\lambda p}^{ \pm} d C_{\mu}(K(n), H)$, and is defined by $(x, y) \rightarrow$ $B_{n}^{ \pm}(\mu,-\nu ; x, y)=\left\langle T_{n}(\mu,-\nu)^{-1} x, y\right\rangle$. There is a unitary action $U$ defined on each of these spaces which is unique and has the property that $d U=d \Pi_{\Lambda}$, with $d \Lambda(H)=\nu+(n-1) / 2$. Moreover,

$$
\begin{aligned}
d \Pi_{\Lambda}(\Omega) & =(s+n / 2)(s-n / 2+1)+\sum_{i=1}^{p-1} \Lambda_{\mu i}\left(\Lambda_{\mu i}+n-2 i-1\right), & & \text { if } n \geqslant 4, \\
& =(s+1) s, & & \text { if } n=2 .
\end{aligned}
$$

8. Concluding remarks. As indicated in the introduction, the irreducible complementary series were first given completely by Knapp and Stein [5]. A different parameter is used in [5], amounting to a change in the logarithmic base, and is given in terms of ours by the formula $(n-1) z / 2=\nu$. The existence of the isolated point and end point representations in the general case seems to be a new result. There are of course some known special cases which we now point out.

For $n=4$, the class of representations given in $\operatorname{IIB}(3)$ correspond to the representations $\Pi_{m 0}$, in Dixmier [2], where $m=\Lambda_{\mu 1}$. Also, the representations in IIB(4), namely $\left[D_{\lambda 2}^{ \pm} d C_{\mu}(K(4), H), d \Pi_{\Lambda}\right]$, correspond to the representations $\Pi_{m,-s}^{\mp}$ in Dixmier's notation, where $m=\Lambda_{\mu 1}$. These facts were already pointed out in [10], although we did not prove unitarizability there for the case of general $n$.

We remark next that the one case of the reducible principal series is contained in the cases $\operatorname{IIB}(4)$. This situation occurs when $\nu=0$, and $\mu$ is a faithful representation of $M(n)$. In this case we have $s=-1 / 2$; so that the projections $D_{\lambda p}^{+}$and $D_{\lambda p}^{-}$have the property that $D_{\lambda p}^{+}+D_{\lambda p}^{-}=1$. The inner products $B_{n}^{ \pm}(\mu, 0 ; \cdot, \cdot)$ reduce to the restrictions of the inner product $\langle\cdot, \cdot\rangle$ to the ranges of these projections. Hence, we have for this case: $L_{\mu}^{2}(K(n), H)=H_{\mu \nu}^{+} \oplus$ $H_{\mu \nu}^{-}$. This is a precise generalization of what happens in the case of $\operatorname{SL}(2, \mathbf{R})$. 
Because of the last remark and because of the formal analogy of the cases IIB(4) to the known cases of $n=4$, and $n=2$, we conjecture that IIB(4) comprises the square integrable representations for $\operatorname{Spin}(1,2 p)$. These square integrable representations are known to exist, because of Theorem 16 in [3c]. We hope to present a proof of this conjecture in a later paper. Knapp and Okamoto [5] have discovered generalizations of the split principal series in the case of the isometry groups of the Hermitian symmetric spaces. IIB(4) provides such a generalization in the direction of the Lorentz groups. A proof of the above conjecture would also provide a similar generalization of the limits of the holomorphic discrete series of Knapp and Okamoto.

For general $n$, the existence of the representations in IIB(1) were pointed out in Takahashi [9], for the special case when $j=1$, and $\Lambda_{\mu}=0$. In the quotient realization of these representations, (Remark 2, of the last section) these representations are the quotient representations of nonunitary principal series by the finite-dimensional representations having $K(n)$-fixed vectors. Takahashi noted in [9] that for $n=3$, these representations are equivalent to certain principal series representations. More precisely, if $j=1, n=3$, the representation in IIB(1) is unitarily equivalent to the irreducible principal series representation $\left[L_{\mu}^{2}(K(3), H)\right.$, $\Pi_{\Lambda}$ ] with $\nu=0, d \Lambda(H)=-1$, and $\Lambda_{\mu 1}=n_{1}+1$. This result can also be verified by computing the eigenvalues of an element in the center of $U(\mathbf{G}(3))$ algebraically independent of $\Omega$, and noting that the $K(3)$-module structures of the two representations are the same.

The last observation of Takahashi brings us to the question of coincidence of the unitary representations listed in Theorem 3. An examination of the $K(n)$ module structure and the eigenvalues of $\Omega$ shows that if there are any coincidences other than those indicated in the statement of Theorem 3, they must occur in the Takahashi representations cited above. It should be possible to settle the question of coincidence then by computing the center of $U(G(n))$ in this case. However, we have not done this yet.

\section{BIBLIOGRAPHY}

1. V. Bargmann, Irreducible unitary representations of the Lorentz group, Ann. of Math. (2) 48 (1947), 568-640. MR 9, 133.

2. J. Dixmier, Représentations intégrables du groupe de De Sitter, Bull. Soc. Math. France 89 (1961), 9-41. MR 25 \#4031.

3a. Harish-Chandra, Representations of a semisimple Lie group on a Banach space. I, Trans. Amer. Math. Soc. 75 (1953), 185-243. MR 15, 100.

3b. Representations of a semisimple Lie group on a Banach space. II, Trans. Amer. Math. Soc. 76 (1954), 26-65. MR 15, 398.

3c. Discrete series for semisimple Lie groups. II. Explicit determination of the characters, Acta Math. 116 (1966), 1-111. MR 36 \#2745. 
4. K. Johnson and N. Wallach, Composition series and intertwining operators for the spherical principal series, Bull. Amer. Math. Soc. 78 (1972), 1053-1059. MR 46 \#9238.

5. A. W. Knapp and E. M. Stein, Intertwining operators for semisimple groups, Ann. of Math. (2) 93 (1971), 489-578.

6. A. W. Knapp and K. Okamoto, Limits of holomorphic discrete series, J. Functional Analysis 9 (1972), 375-409. MR 45 \#8774.

7. G. Mackey, Induced representations of locally compact groups. I, Ann. of Math. (2) 55 (1952), 101-139. MR 13, 434.

8. G. Schiffmann, Intégrales d'entrelacement et fonctions de Whittaker, Bull. Soc. Math. France 99 (1971), 3-72.

9. R. Takahashi, Sur les représentations unitaires des groupes de Lorentz généralisés, Bull. Soc. Math. France 91 (1963), 289-433. MR 31 \#3544.

10. E. Thieleker, On the quasi-simple irreducible representations of the Lorentz groups, Trans. Amer. Math. Soc. 179 (1973), 465-505.

11. N. Wallach, Kostant's $p^{\gamma}$ and $R^{\gamma}$ matrices and intertwining integrals, Proc. Sympos. Pure Math., vol. 26, Amer. Math. Soc., Providence, R. I., 1974, pp. 269-274.

DEPARTMENT OF MATHEMATICS, UNIVERSITY OF SOUTH FLORIDA, TAMPA, FLORIDA 33620 